\title{
Radio frequency over fiber technology for SKA-low receiver
}

\author{
Federico Perini $\odot,{ }^{\text {a,* }}$ Simone Rusticelli, ${ }^{a}$ Marco Schiaffino, ${ }^{\text {a }}$ \\ Andrea Mattana $\odot,{ }^{a}$ Jader Monari, ${ }^{a}$ Giovanni Tartarini, ${ }^{b}$ Jacopo Nanni $\odot,{ }^{b}$ \\ Budi Juswardy $\odot,{ }^{c}$ Randall Wayth $\odot,{ }^{c}$ and Mark Waterson ${ }^{\mathrm{d}}$ \\ ${ }^{a}$ Istituto Nazionale di Astrofisica, Istituto di Radioastronomia, Medicina, Italy \\ 'Università di Bologna, Dipartimento di Ingegneria dell'Energia Elettrica e dell'Informazione \\ "Guglielmo Marconi," Bologna, Italy \\ ${ }^{\mathrm{c}}$ Curtin University, International Centre for Radio Astronomy Research, Technology Park, \\ Bentley, Australia \\ ${ }^{\text {d} S K A ~ O r g a n i s a t i o n, ~ J o d r e l l ~ B a n k, ~ L o w e r ~ W i t h i n g t o n, ~ M a c c l e s f i e l d, ~ C h e s h i r e, ~ U n i t e d ~ K i n g d o m ~}$
}

\begin{abstract}
The signal reception chain is an essential element for achieving the square kilometer array-low (SKA-low) system requirements in terms of high sensitivity and dynamic range. The balance between gain, linearity, and low power consumption, as well as the cost, are fundamental parameters that influence the selection of the most suitable technology for SKA-low. Further factors, such as low self-generated radio frequency (RF) interference, high reliability, robustness under extreme environment, and last but not least, the distance between the antennas and the acquisition systems, have impacts on the selection for both architecture and receiver system design. The selected technology for the SKA-low RF signal transportation is RF-over-fiber systems, where the preamplified RF signal picked up by the antennas is carried via analogue modulation over optical fiber. The rationales behind the selection are reported, along with descriptions on the development of the receiver prototypes. The prototypes were deployed and installed on the demonstrator arrays at the selected SKA-low site in Western Australian. Particular attention has been put on the thermal characterization of the receiver system under the actual operating temperature on site, especially when both transmitting part and the optical medium are subjected to external ambient temperature variations. Performance issues encountered in the demonstrator arrays are also discussed along with some proposals for future activities. $\odot$ The Authors. Published by SPIE under a Creative Commons Attribution 4.0 International License. Distribution or reproduction of this work in whole or in part requires full attribution of the original publication, including its DOI. [DOI: 10.1117/1.JATIS.8.1.011016]
\end{abstract}

Keywords: radio frequency-over-fiber; antenna array; C-wavelength division multiplexing; light amplification by stimulated emission of radiation; low-frequency receiver; SKA-low.

Paper 21097SS received Aug. 20, 2021; accepted for publication Dec. 22, 2021; published online Jan. 11, 2022.

\section{Introduction}

Analogue radio frequency-over-fiber (RFoF) technology is widely used in various industries from cable television (CATV) and mobile telecommunications to radio astronomy instrumentation. This technology has been used for many years to transfer TV signals via intermediate and long-haul links, exploiting the advantages connected to the use of optical fibers like low attenuation, high transmission bandwidth, low cable dimensions, and immunity to electromagnetic interference. Furthermore, in recent years, RFoF has revolutionized the mobile telecommunications industry, especially for giving mobile users full coverage on radio-shadowed areas such as road tunnels, underground stations, or large/high buildings. These links have been deployed in their millions in mobile networks in developing countries, such as China, as a cost-effective way to expand the coverage of base stations. As a result of such mass production and improved production facilities, RFoF links employing high-performance lasers, such as distributed

*Address all correspondence to Federico Perini, federico.perini@inaf.it 
feedback (DFB) ones, and detectors have reduced dramatically in cost, and the whole industry sector has become established.

Within the described context, the challenging requirements associated with the realization of the square kilometer array $\left(\mathrm{SKA}^{1}\right)$, and particularly with the first phase of its low-frequency band coverage version (SKA-low) can be adequately met exploiting the maturity and the effectiveness of the RFoF technology.

Indeed, SKA-low, which will operate in the bandwidth 50 to $350 \mathrm{MHz}$, will consist of 131,072 antennas, which will be deployed in the Shire of Murchison in Western Australia. The extreme environmental conditions, combined with the limited presence of pre-existing services and facilities of the location, pushed since the beginning to envisage the realization of a single central processing facility (CPF), performing signal digitization, preprocessing, and final processing, plus a relatively limited number of remote processing facilities (RPFs) performing signal digitalization and preprocessing, connected through a standard digital transmission system to the CPF for final processing. CPF and RPFs will all be equipped to operate adequately in such a hostile environment and will be directly connected, respectively, to about $60 \%$ and $40 \%$ of the antennas. It is, therefore, necessary to ensure the delivery of the radioastronomical signals with the required degree of fidelity for distances between antennas and CPF/RPFs, which can range from around $500 \mathrm{~m}$ to few kilometers.

The choice of the optical fiber as transmission channel results then to be the only one that allows compliance with the mentioned constraints, and in the final design of SKA-low, the radioastronomical signals coming from 512 array stations each one consisting of 256 double polarization antennas will be connected either to the CPF or to the correspondent RPF through an appropriate RFoF-based link. ${ }^{2}$

The structure of the RFoF receiver to be utilized in SKA-low, which is to date almost completely finalized, is the result of successive design and refinement operations that took place in the latest years thanks to the realization of demonstrator stations. Two examples of these SKA-low demonstrators, all deployed at the Murchison Radio-astronomy Observatory (MRO) in the immediate vicinity of the actual SKA-low location, are given by the aperture array verification system and the engineering development array, in their different phases (AAVS $1,{ }^{3}$ $\mathrm{AAVS} 2{ }^{4}$ and EDA $1,{ }^{5} \mathrm{EDA} 2,{ }^{6}$ respectively). Each one of these demonstrators has the size of one SKA-low array station, i.e., it consists of 256 double polarization antennas, SKA log periodic antenna (SKALA2 and SKALA4.1) for AAVS systems, and Murchison widefield array $\left(\mathrm{MWA}^{7}\right)$ dipole for EDA systems. The stations are connected via RFoF-based links of about $5.5 \mathrm{~km}$ length to a shielded room borrowed from the Australian square kilometer array pathfinder $\left(\mathrm{ASKAP}^{8}\right.$ ) facility, where all the CPF functionalities are performed.

In this work, the structure of the SKA-low RFoF-based receiver resulting from the mentioned engineering development and design activities will be illustrated, also describing physical aspects that motivated the technological solutions adopted.

This paper is organized as follows. In Sec. 2, the choice of the RFoF technology for a category of radio astronomical facilities will be technically motivated, and some general features of the SKA-low RFoF-based receiver will be illustrated. Section 3 will describe the receiver realization and characterization, both in its electronic and optical aspects, whereas Sec. 4 will be devoted to some topics that are still under investigation and give room for further improvements in view of the ultimate receiver realization. Finally, conclusions will be drawn in Sec. 5.

\section{RFoF Technology in Radio Astronomy and in SKA-low}

For most radio astronomy applications, the transfer of the signal from the antenna to the receiver and processing system has been implemented using coaxial connections. Coaxial cables can be made to work up to $60 \mathrm{GHz}$, however, RF loss and frequency-dependent loss (slope), specially for multioctave bandwidth systems, are significant and limit the maximum length for their use. Therefore, optical fiber becomes an attractive alternative for all systems where distance and/or $\mathrm{RF}$ bandwidth to be transmitted become significant.

The principle of using RFoF for radio astronomy is not a novel one. As the RFoF technology has become more mature, it has gradually replaced more and more the coaxial links used in the

J. Astron. Telesc. Instrum. Syst. 011016-2 Jan-Mar 2022 • Vol. 8(1) 
Table 1 Different radio astronomical facilities formed by a high number of antennas, operating at frequencies below $2 \mathrm{GHz}$ and techniques utilized to realize their respective antenna-to-processing room down links (DL).

\begin{tabular}{lcccl}
\hline \hline Name & Location & Bandwidth (MHz) & DL length & DL technology \\
\hline CHIME $^{15}$ & Canada & 400 to 800 & $50 \mathrm{~m}$ & RF over Coax \\
HIRAX $^{16}$ & South Africa & 400 to 800 & Up to $1 \mathrm{~km}$ & RFoF \\
GMRT $^{17}$ & India & 50 to 1500 & Up to $20 \mathrm{~km}$ & RF/IF over fiber \\
HERA $^{18}$ & South Africa & 100 to 200 & 150 to $500 \mathrm{~m}$ & RF over Coax/RFoF \\
LOFAR $^{19}$ & Netherlands & 10 to 250 & $200 \mathrm{~m}$ & RF over Coax \\
MWA $^{20}$ & Australia & 80 to 300 & $5 \mathrm{~km}$ & RF over Coax/RFoF \\
OVRO LWA $^{21}$ & USA & 27 to 85 & Up to few km & RF over Coax/RFoF \\
SKA-LOW & Australia & 50 to 350 & $6 \mathrm{~km}$ & RFoF \\
\hline \hline
\end{tabular}

existing radio telescopes since their construction, to carry both RF/IF and synchronization signals (from few $\mathrm{MHz}$ to several $\mathrm{GHz}$ ), as happened for example to the Italian radiotelescopes such as the Northern Cross ${ }^{9}$ and the Medicina VLBI dish. ${ }^{10}$ However, its adoption in large low-frequency arrays, both in terms of number of antennas/receivers and their physical extension, has been studied and debated in the radio astronomical community for at least two decades, ${ }^{11-14}$ because the coaxial links could continue to represent an attractive alternative. A universal solution does not exist and for each system the decision has to be taken considering several factors such as its location, architecture, cost cap, reliability, and maintainability along with the requested RF performances. As a confirmation of this point, Table 1 allows to appreciate the manifold solutions presently adopted to realize the antenna downlinks of some of the main radio astronomical facilities operating at frequencies below $2 \mathrm{GHz}$.

As representative example of the fact that the appropriate technology can vary in relation to the characteristics of the facility considered, Fig. 1 compares the array configurations where extensively distributed RPFs collect the signals coming from very closely located antennas [this is the case of the low-frequency array radiotelescope (LOFAR)], and where a relatively low number of RPFs collect the signals coming from the subset of antennas located in their vicinity

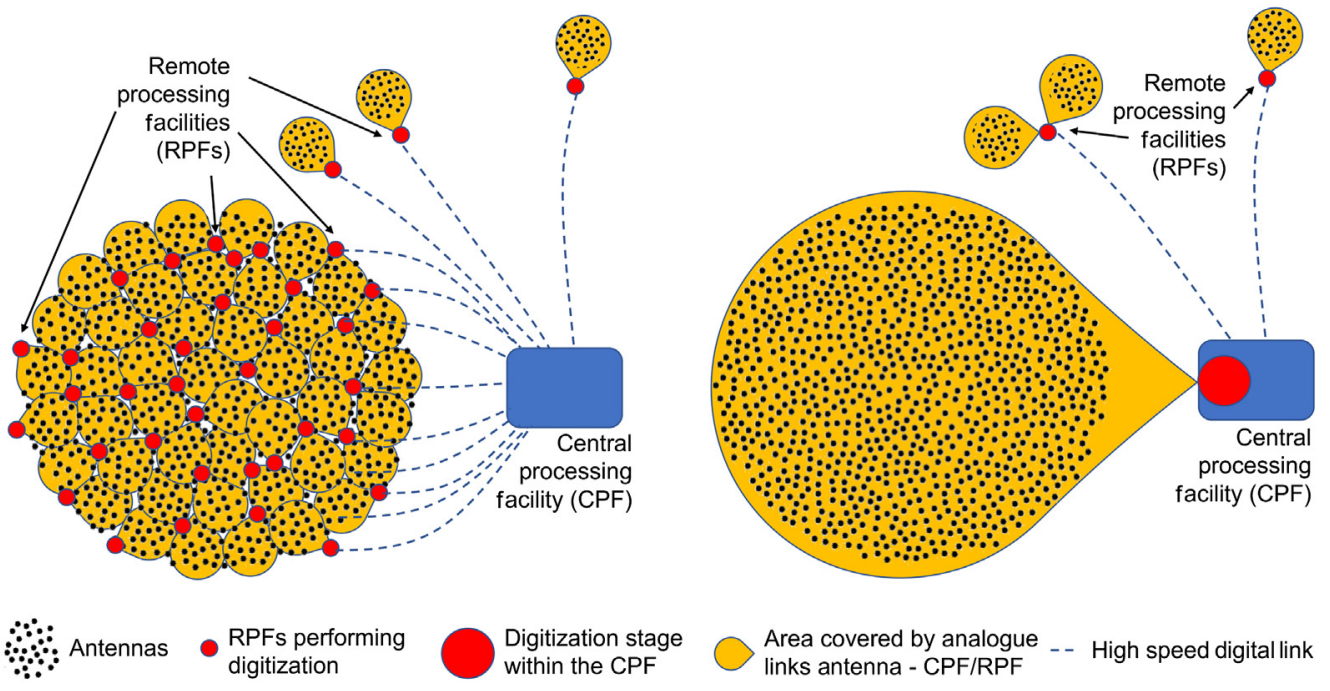

Fig. 1 Examples of different array configurations. (a) LOFAR: all antennas are connected to extensively distributed RPFs. (b) SKA-low: a subset of antennas is connected to a relatively low number of RPFs while their major part is directly connected to the CPF. 
while at the same time a single CPF collects the signals coming from the major part of the antennas, with distances that can reach the value of some kilometers (this is the case of the SKA-low radiotelescope).

For the first configuration, the well-established 75-Ohm coax cable for TV signal distribution networks widely utilized as antenna cable for terrestrial and satellite broadcast systems has been chosen due to its intrinsic low cost and durability. But even if LOFAR maximum frequency is quite low (290 MHz), a few hundred meters long cables would already introduce a too large gain variation over the band, which limits the dynamic range and requires extra circuits to compensate for it. For that reason, the cable length between the antennas and their receivers is kept below $200 \mathrm{~m}$ and one RPF is required for each single station. On the contrary, for SKA-low, as mentioned in Sec. 1, a different choice for the array topology had to be made due to the constraints of the Shire of Murchison environment, in which relatively few RPFs will collect the radioastronomical signals from around 200 stations of 256 antennas each, located at distances of around $500 \mathrm{~m}$ from the respective RPFs, whereas the CPF will directly receive the signals coming from the remaining around 300 stations of 256 antennas each, with connections that can reach lengths of a few kilometers.

If the frequency $f_{\mathrm{RF}}$ of the signal to be transmitted is not very high (up to some $\mathrm{GHz}$ ), $\mathrm{RFoF}$ links can be realized exploiting the so-called direct intensity modulation-direct detection technique (direct IM-DD). Employing such technique, the input RF signal $I_{\mathrm{RF}, \mathrm{TX}} \cos \left(2 \pi f_{\mathrm{RF}}\right)$ is superimposed to the laser bias current, generating a dynamic component of the optical power $P_{\text {opt }}(t)=\eta_{\mathrm{RF}} I_{\mathrm{RF}, \mathrm{TX}} \cos \left(2 \pi f_{\mathrm{RF}}\right)$, where $\eta_{\mathrm{RF}}$ is the modulation response coefficient of the laser, with an order of magnitude roughly around $0.1 \mathrm{~mW} / \mathrm{mA}$. After propagating in the optical fiber span of length $L$ with an attenuation $A_{\text {opt }}=10^{\alpha L / 10}$ (with $\alpha$ attenuation coefficient of the optical power, equal to around $0.4 \mathrm{~dB} / \mathrm{km}$ for systems operating at a wavelength $\lambda \approx 1.3 \mu \mathrm{m}$ ), the signal is finally back converted into an RF current by the photodiode (PD) through the responsivity coefficient $\mathcal{R}$, whose order of magnitude is roughly around $1 \mathrm{~mA} / \mathrm{mW}$. A passive match to $50-\mathrm{Ohm}$ impedance is then typically present, which further reduces by a factor of two the final current made available at the back end. The amplitude of the RF received signal can then be taken as the amplitude of the input signal $I_{\mathrm{RF}, \mathrm{TX}}$ multiplied by the coefficient $g=\eta_{\mathrm{RF}} \cdot 10^{-\frac{\alpha L}{10}} \cdot \mathcal{R} / 2$.

Considering the output and input RF powers, which are proportional to the square of the correspondent current amplitudes, their ratio results instead given by $G=g^{2}$, bringing to an RF power attenuation of about $26 \mathrm{~dB}$, only weakly influenced by the value of $L$.

This means that the low attenuation of the optical fiber (which, if the RF powers are concerned, is proportional to $10^{\frac{2 \alpha L}{10}}$, i.e., grows with a coefficient of $2 \cdot \alpha=0.8 \frac{\mathrm{dB}}{\mathrm{km}}$ ) can show all its potential only above a certain distance, making RFoF advantageous with respect to coaxial ones.

As mentioned above, considering the SKA-low array layout, the minimum link length will be about $0.5 \mathrm{~km}$ for around $40 \%$ of the antennas. As can be appreciated in Fig. 2, such distance already gives attenuations of more than $50 \mathrm{~dB}$ using the coaxial cables adopted by LOFAR (Coax-9), considering the upper portion of the frequency band. The value of the attenuation

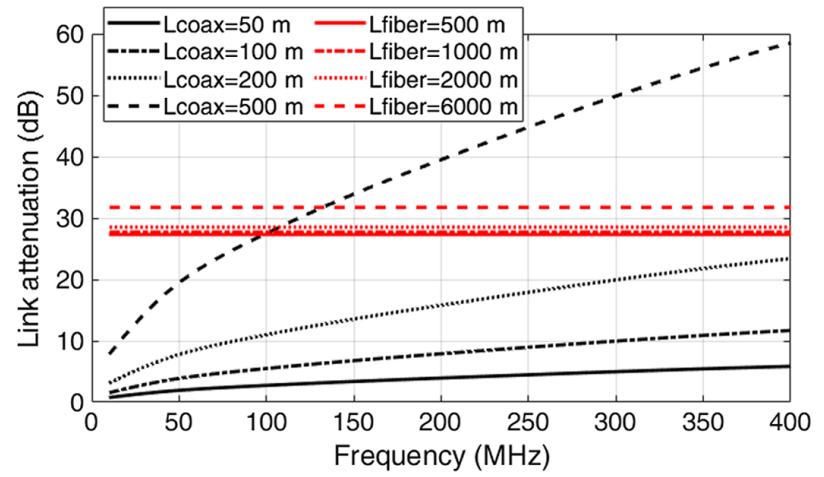

Fig. 2 Attenuation for both coaxial cables (black traces) and RFoF links (red traces) versus RF frequency and cable/link length. 
is then already very high at this distance and would become completely unacceptable for the links of higher lengths.

The use of the coaxial cable had therefore to be excluded, while, at the same time, the characteristics of the optical fiber made it possible the design of SKA-low with the required topology. Indeed (see again Fig. 2), thanks to the low value and the frequency flatness of the optical fiber attenuation coefficient, all antennas of the array will be efficiently connected to the respective processing units with just a few $\mathrm{dB}$ of loss difference between the shorter and the longer optical links in all the portions of the 50-350 $\mathrm{MHz}$ frequency band.

Once the choice of the RFoF technology was made, a further decision had to be taken with respect to the kind of laser on which to realize the optical transmitter. Also exploiting a detailed experimental characterization of different kinds of lasers, DFB ones were finally chosen. Indeed, transmitters based on vertical cavity surface emitting laser (VCSELs), typically exhibit higher values of distortion and noise, though presenting attractive features in terms of low cost and lowpower consumption. ${ }^{23-25}$ Those based on Fabry-Perot (FP) lasers tend instead to present higher noise and comparable/lower distortion performances with respect to DFB-based ones ${ }^{26,27}$ and could represent a possible solution in case their cost were substantially lower. However, only FP lasers working at the wavelengths of 1310 and $1550 \mathrm{~nm}$ can be effectively regarded as very lowcost devices, because of their popularity on the market. On the contrary, to have available FP lasers emitting at other wavelengths-like the ones at 1270 and $1330 \mathrm{~nm}$ that, as will be illustrated below, had to be utilized for the SKA-low RFoF link realization-would require high expenditures due to the necessity to customize chips. Taking then into account the fact that in the latest years commodity applications, such as fiber-to-the-home, have driven for DFB lasers the volumes up and consequently the costs down to a few tens of euros each (in high volumes), the choice of DFB lasers was the most appropriate in terms of all the aspects considered.

More in general, the mentioned cost reduction regards a number of key optical components (photodiodes and optical filters), making the utilization of high-quality devices that typically work up to a few $\mathrm{GHz}$ relatively affordable, and this allowed the customized design of the SKA-low receiver system based on RFoF low-frequency links. Indeed, commercial-off-the-shelf available RFoF links, which feed remote wireless transceivers and other antenna systems for communication (satellite and mobile phone antennas up and downlinks) and CATV, are typically more complex and not optimized for the SKA-low band where, even if the maximum frequency is quite low, the cost is the main constraint. Summarizing, the commercial availability of the components required allowed to build a customized, minimum complexity link suitable for SKA-low.

The first generation of RFoF links for the analogue transmission to the CPF was based on standard DFB laser working at $1310 \mathrm{~nm}$, with one laser/one fiber for each antenna polarization (see upper part of Fig. 3).

To reduce the infrastructural cost, wavelength division multiplexing (WDM) technology has been adopted for the analogue transmission of the two antenna polarizations to the CPF. The WDM technique is widely used in fiber optic communication systems. The idea is to utilize the same optical fiber for the simultaneous transmission of different signals, and each one is used to modulate a different optical carrier at a different wavelength. Different carriers do not interfere during propagation, and they can be easily demultiplexed at the receiver side, allowing the signals to be processed separately. The adoption of WDM for SKA-low is possible because only two signals must be propagated on the same fiber, which means that cheaper coarse-WDM (C-WDM) components can be used instead of the more expensive dense-WDM (D-WDM) ones. For C-WDM, the separation between the different wavelengths is larger than in the D-WDM, which means that it is possible to use simpler and hence cheaper optical devices (i.e., optical duplexers). In this case, the foreseen advantages are related to the global cost and complexity reduction of the optical infrastructure thanks to the halving of the number of fibers, optical connectors, and fusion splices (see bottom part of Fig. 3).

As mentioned, for SKA-low, two wavelengths are multiplexed, carrying, respectively, the horizontal and the vertical polarizations of the received radio-astronomical signal from the field. The choice of the wavelengths has been made to have an acceptable optical attenuation and at the same time avoid strong phase difference (named also phase error) among different optical links of km-range carrying the same $\lambda$. This last phenomenon depends on the effects of fiber chromatic

J. Astron. Telesc. Instrum. Syst. $\quad$ 011016-5 Jan-Mar 2022 • Vol. 8(1) 


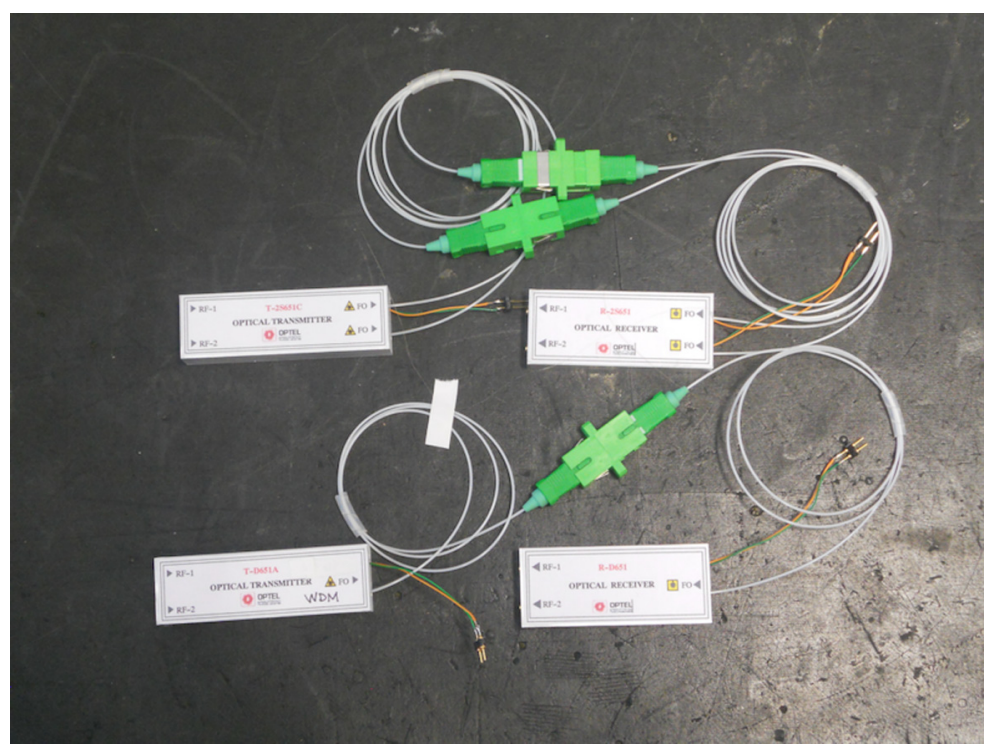

Fig. 3 Standard (upper) and WDM (lower) RFoF transmitter-receiver pair prototype developed by IRA/INAF for SKA-low.

dispersion and temperature-induced wavelength shift, both depending on the wavelength (see Secs. 3.2.1 and 3.2.2).

Finally, note that the typical problem of the fiber nonlinear effects is expected to be absent in the case of SKA-low, since the levels of the optical power carried by the fiber will be much lower than in the case typical of the digital transport optical network, where several optical carriers are transmitted with the D-WDM technology.

\section{SKA-Iow Receiver Design and Analysis}

There are several factors that need to be considered carefully to evaluate the feasibility of RFoF for radio astronomy applications. The most important parameters being considered are gain and phase stability over temperature (contribution from both the electronics in the optical transmitter and the fiber optic cable), since this has a major effect on the performance of the instrument and how frequent calibration needs to be performed.

These aspects of the receiver for SKA-low have been studied through a few consortium projects, such as the Aperture Array Verification Program (AAVP) and the Aperture Array Design Consortium (AADC), where colleagues from different institutions as the Dutch Institute for Radio Astronomy (ASTRON ${ }^{28}$ ), the Australian International Centre for Radio Astronomy Research $\left(\mathrm{ICRAR}^{29}\right)$, the University of Cambridge $\left(\mathrm{UCAM}^{30}\right)$, the Italian National Institute for Astrophysics (INAF ${ }^{31}$ ), and University of Bologna (UNIBO) ${ }^{32}$ have analyzed all these specific issues and subsequently verified and improved the RFoF module designed for the prototypes/ demonstrators mentioned in Sec. 1, in particular AAVS1, AAVS2 (see Figs. 4(a) and 4(b), respectively), and EDA2, filling those design gaps found during the field campaign tests.

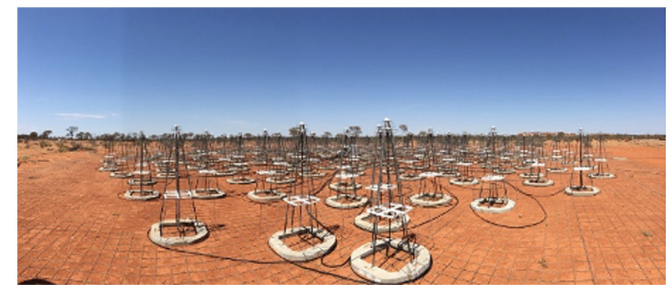

(a)

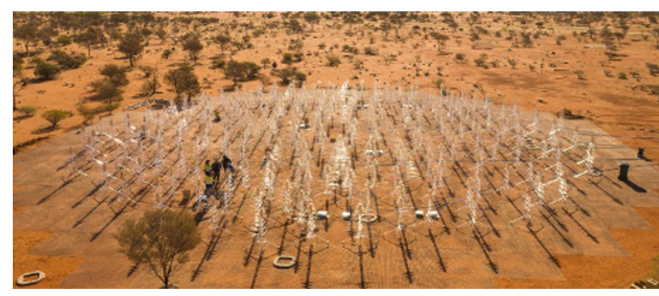

(b)

Fig. 4 (a) AAVS1 and (b) AAVS2 prototype stations deployed at the MRO, Western Australia. 
Members of the receiver task group have carried out measurement campaigns to evaluate the performance of RFoF links for SKA-low, for three prototypes made by ASTRON, INAF, and UCAM within laboratories setup. After testing the RF performance of the proposed systems, the INAF design was chosen to be adopted in AAVS1 and in the following systems as it was more mature than the other prototypes.

INAF RFoF technology is fully integrated within the SKA-low analogue receiver chain that performs the following functions:

- amplifies the signals collected from each antenna to a level suitable to be converted by the analogue to digital converter (ADC);

- filters the received band, to avoid aliasing on the high portion of the spectrum, and rejecting RFI outside the band of interest;

- transports the signal from the field node (station) to the acquisition system.

\subsection{Structure of the Receiver and Tests of Compliance with SKA-Iow Specifications}

The analogue receiver is divided into three main parts: the RF/optical transmitter, called front end module (FEM), the optical fiber, and the optical/RF receiver, called preanalogue to digital unit (PREADU). The RFoF link is completely embedded in the RF analogue receiver chain since (see Fig. 5) its active components, the WDM laser sources and the WDM double PD, are parts of the FEM and PREADU circuits, respectively.

Two FEM installation options have been investigated and tested in the field thanks to the demonstrators. For AAVS1 [see Fig. 6(a)], FEMs are located within the antennas apex, just after the low-noise amplifier (LNA) and connected by a hybrid fiber-optic and copper power cable to a central antenna power interface unit (APIU). For AAVS2 [see Fig. 6(b)], the FEMs for a group of antennas are installed inside a box called small modular aggregation and RFoF trunk box (SMARTbox) and their connection with the antennas is by short $(\sim 10 \mathrm{~m})$ coaxial cables. The current design with SMARTbox for SKA1, implemented and tested with AAVS2, offers advantages for maintainability and accessibility with respect to the original concept adopted in AAVS1.

The FEM contains a high-pass filter, with a 3-dB cut-off frequency around $35 \mathrm{MHz}$, which helps to reject strong radio frequency interferers (RFI) located within its stop band, the in-line bias-T to feed the LNA power, an RF amplifier, and the laser with its automatic optical power control circuit. As depicted in the general scheme of Fig. 5, the 2 polarization signals directly modulate the laser currents operating at 1270 and $1330 \mathrm{~nm}$, which are multiplexed onto a single optical fiber for each antenna and then routed from the field node to the remote acquisition systems. Figure 7 allows to appreciate the actual realization of the FEM and of the PREADU.

At the processing facility, the PREADU board converts the optical signal back to RF using a WDM double PIN photodiode receiver, which separates the two RF polarizations, performs low-pass filtering for antialiasing, and provides further amplification prior to the ADC. A digital

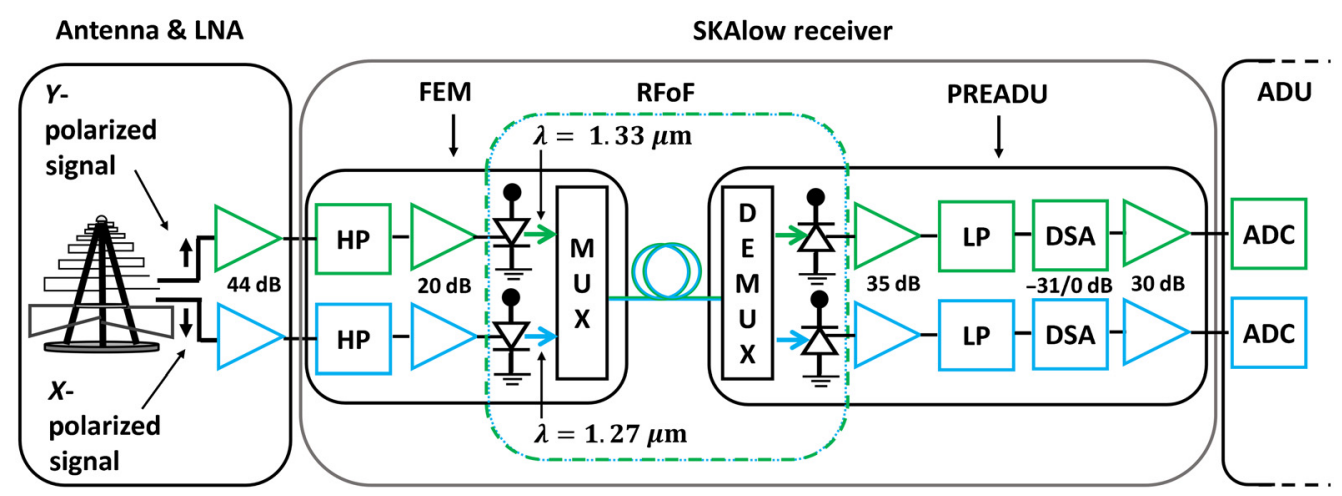

Fig. 5 SKA-low receiver chain. 


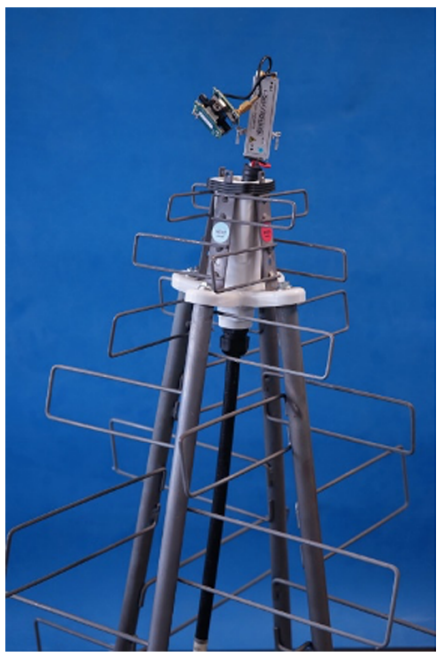

(a)

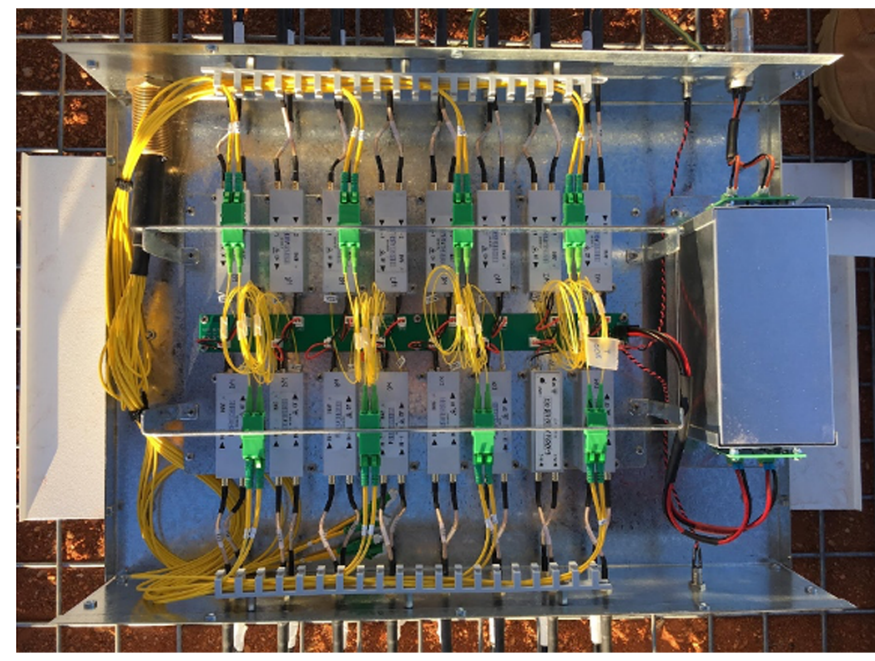

(b)

Fig. 6 FEM installation options: at the SKALA2 antenna apex for (a) AAVS1 and grouped by 16 inside the SMART boxes for (b) AAVS2.

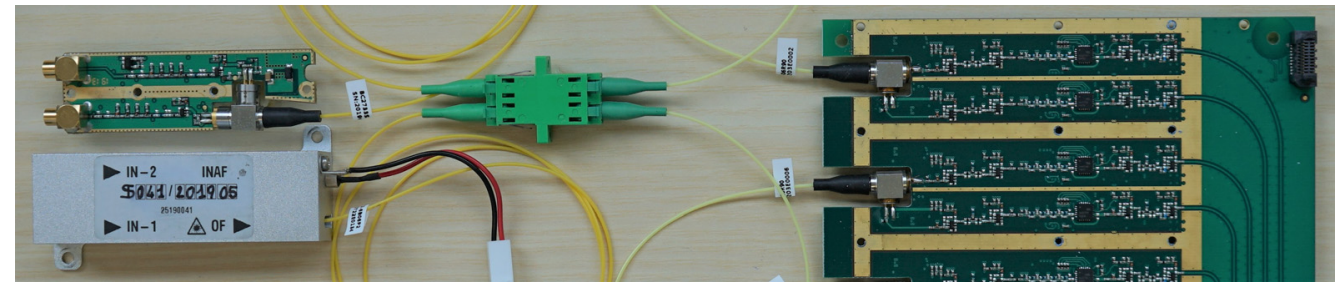

Fig. 7 FEM PCB and its enclosure on the left and a portion of a PREADU board with the metallic shield removed on the right.

step-attenuator (DSA) and a final amplification stage set the correct levels to the acquisition system (see again Fig. 7 on the right).

In the current design, 32 signal RF chains will be assembled in two "receiver module" (PREADU) each carrying 16 channels, the two RF polarizations of 8 antennas [see Fig. 8(a)]. Receiver modules are mounted on the signal processing system processing boards (ADU board), containing the ADCs and the digital components. One ADU board and two PREADU boards compose a tile processing module (TPM), which is shown in Fig. 8(b). Each station includes 16 TPM modules and supporting systems, hosted in a temperature controlled and screened facility: the CPF, for all the core stations, or the RPFs, for stations in the array arms, to limit maximum fiber length.

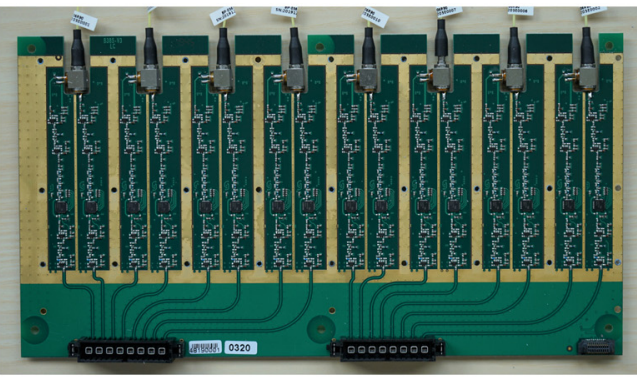

(a)

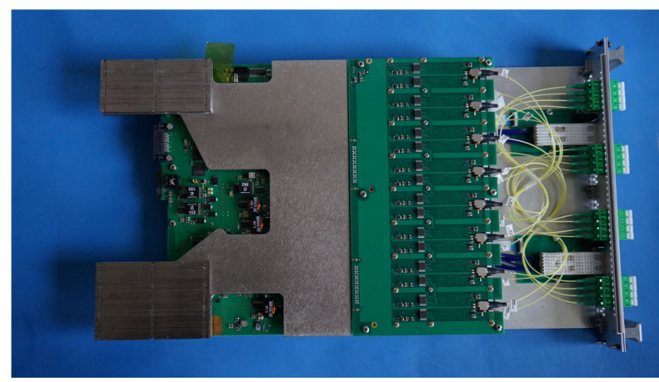

(b)

Fig. 8 (a) One PREADU board and (b) an assembled TPM where two PREADU boards are connected to an ADU board. 
From the analysis point of view, the derivation of the main receiver specifications from SKA's level 2 (L2) requirements was found to be a crucial aspect. Some boundary conditions have been assumed, such as the performances of the antenna/LNA, the main ADC specs (i.e., number of bits, Vpp,...), and the RFI measured at the MRO site. With regards to the data acquisition part and to the evaluation of the optimal RF level at the input of the sampler delivered from the analogue receiver, the analysis carried out also considers the correlation systems that exist in the digital section, in particular, the requirements for:

1. high dynamic range, for accurate and deep sky mapping, SKA high-level specification requires the system to be able to accurately map sources with a flux $50 \mathrm{~dB}$ below those of the large sources in the field of view;

2. high linearity (low intermodulation products), to a level at least below the instantaneous noise level in the fine channelization bandwidth $(\approx 1 \mathrm{kHz})$ of the correlator;

3. low added noise (quantization noise $<0.5 \%$ of the total).

The conclusions of the analysis are the SKA-low receiver requirements reported in Table 2, where only the ambient (air) temperature range at MRO has been specified as $-10^{\circ} \mathrm{C}$ to $+50^{\circ} \mathrm{C}$. Note that the real operative temperature of the electronics composing the receiver chain and, in particular, of the FEM, strongly depends on its location. Measurements carried on with SKALA2/AAVS1 system showed that the maximum operative temperature experienced by the FEM is about $+70^{\circ} \mathrm{C}$, similar situation with the first release of the AAVS2 SMART boxes. In both cases, the actual temperature reached by the FEM printed circuit board (PCB) is even in the excess of $+80^{\circ} \mathrm{C}$. Such high temperatures cannot be tolerated, both for the detrimental effects on the behavior of the electronics and for the expected reduced lifetime of the devices, especially the laser sources. For that reason, a particular attention has been devoted to optimize the thermal aspects of the FEM installation, which

Table 2 SKA-low receiver specifications (second column) and the correspondent FAT requirements (third column).

\begin{tabular}{|c|c|c|}
\hline \multirow[b]{3}{*}{ Parameter } & "SKA-low receiver requirements & FAT requirements \\
\hline & Temp range: $-10 /+50^{\circ} \mathrm{C}$ & Room temperature $\left(23^{\circ} \mathrm{C}\right)$ \\
\hline & FEM/PREADU direct connection & Fiber length up to $6 \mathrm{~km}$ \\
\hline Frequency range & \multicolumn{2}{|c|}{50 to $350 \mathrm{MHz}$} \\
\hline Instantaneous bandwidth & \multicolumn{2}{|c|}{$300 \mathrm{MHz}$} \\
\hline Return loss & \multicolumn{2}{|c|}{$>12 \mathrm{~dB}$} \\
\hline Nominal gain & $41 \mathrm{~dB}$ & $\begin{array}{c}\min 54 \mathrm{~dB}, \text { typ } 60 \mathrm{~dB} \\
\max 66 \mathrm{~dB}\end{array}$ \\
\hline Gain flatness & \multicolumn{2}{|c|}{ $\pm 1.5 \mathrm{~dB}$} \\
\hline Out-of-band suppression & \multicolumn{2}{|c|}{$>50 \mathrm{~dB}$ for $f<20 \mathrm{MHz}$ and $f>450 \mathrm{MHz}$} \\
\hline $\mathrm{RF}$ isolation RF1-RF2 & \multicolumn{2}{|c|}{$>30 \mathrm{~dB}$} \\
\hline NF & $<22 \mathrm{~dB}$ & $<16 \mathrm{~dB}$ \\
\hline $1 \mathrm{~dB}$ compression point & \multicolumn{2}{|c|}{ OP1 $\mathrm{dB}>+17 \mathrm{dBm}$ at $100 \mathrm{MHz}$} \\
\hline Second-order intermodulation & \multicolumn{2}{|c|}{ OIP2 > +38 dBm } \\
\hline Third-order intermodulation & \multicolumn{2}{|c|}{ OIP3 $>+18 \mathrm{dBm}$} \\
\hline Power consumption & $<0.65 \mathrm{~W}$ for $\mathrm{FEM}$ & $<0.55 \mathrm{~W}$ for FEM \\
\hline & $<1.35 \mathrm{~W}$ for $1 / 8$ of PREADU & $<1.32 \mathrm{~W}$ for $1 / 8$ of PREADU \\
\hline
\end{tabular}




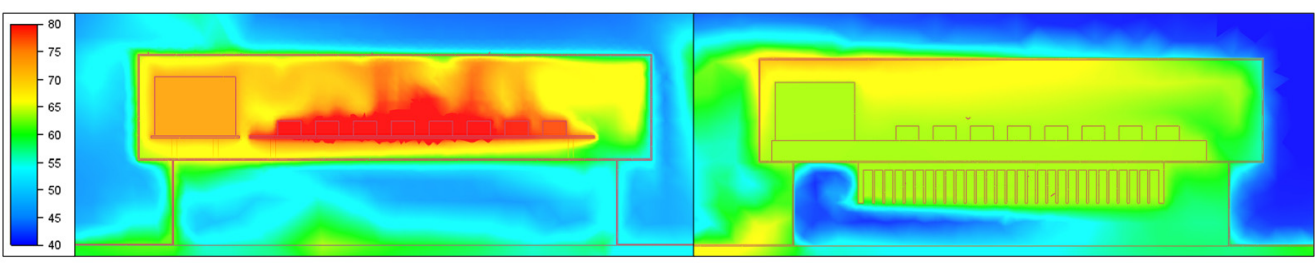

(a)

(b)

Fig. 9 (a) Thermal simulation of the first and (b) the optimized AAVS2 SMARTbox model. The following boundary conditions have been considered: external temperature: $42^{\circ} \mathrm{C}$, solar thermal flux: $2019 / 12 / 10,02: 30 \mathrm{pm}$, latitude: $-26^{\circ} 42^{\prime}$, longitude: $116^{\circ} 40^{\prime}$. Temperature scale is from $+50^{\circ} \mathrm{C}$ to $+80^{\circ} \mathrm{C}$.

has led to the last SMARTbox design where the maximum operative temperature has been reduced to $+65^{\circ} \mathrm{C}$ (see Fig. 9).

Considering the large numbers of receiving chains (Fig. 10) and the impracticality to test all the electronics for the whole set of the specifications (for the full range of FEM temperatures and different lengths of fiber), the correspondent factory acceptance test (FAT) performed at ambient temperature and FEM-PREADU direct connection has been derived.

Below are reported both some test examples of receiver specs verifications (see Fig. 11) and an example of the results of the FAT measurements that were performed for the entire production for AAVS1, EDA2, and AAVS2 (see Figs. 12 and 13).

\subsection{Gain and Phase Response of the Receiver Under Temperature Variations}

Science data processing and analysis techniques require the signal chains to be stable across all of the data samples collected in an observation so that resulting variations in the data output can be attributed to the observed sky and not to the measurement system. Calibration of the signal chains is the adjustment of the signal chains to standardize and hopefully optimize their behavior at a point in time, and the system is required to maintain this behavior between reference measurements, optimally for long periods to ensure that data are consistent and to minimize the overhead in capturing and processing calibration data.

Practical calibration strategies basically compare the response of all signal chains in a system to a common reference signal and adjust or offset their actual complex transfer functions to achieve a desired, or at least similar, gain and phase behavior at all frequencies across the band. Such reference measurements can only be done when suitable input signals are available. Because of the large number of independent signal inputs, local reference signal generation was deemed too expensive to implement at the required level of accuracy. However, the sky itself presents a common input signal to all antennas that can be used as the calibration reference with appropriate processing. Decomposing the transfer function into terms related to individual
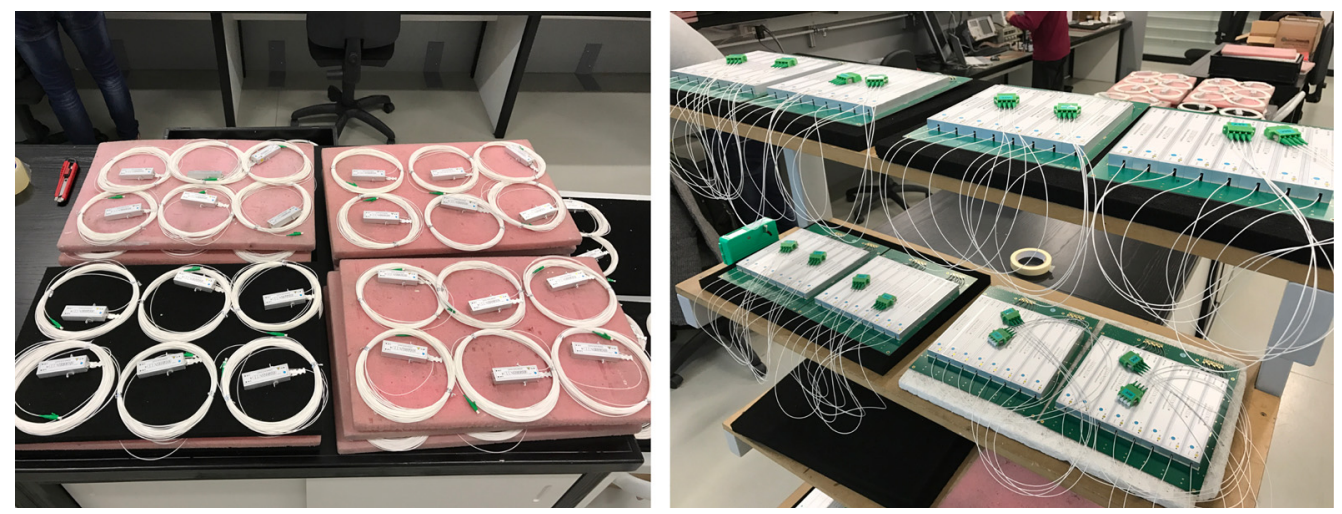

Fig. 10 Pictures taken during the AAVS1 FEM and PREADU productions. 


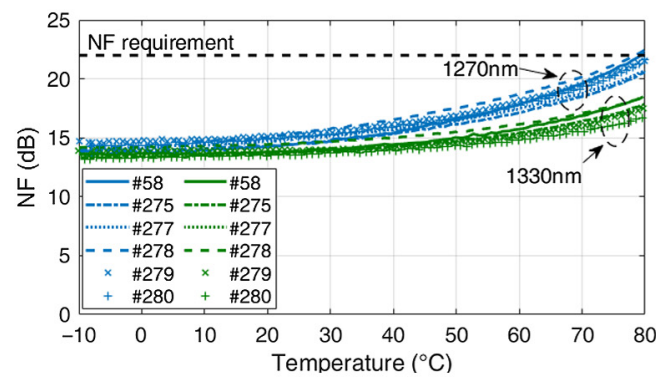

(a)

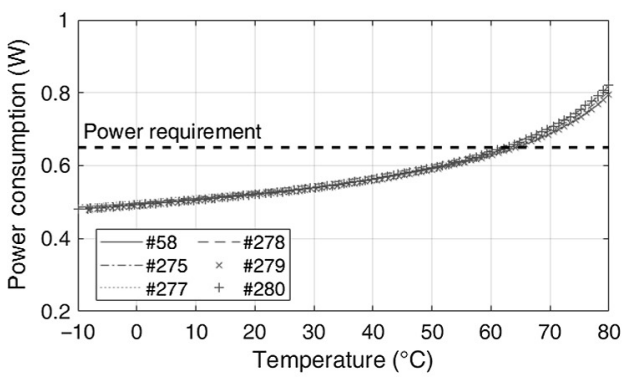

(b)

Fig. 11 Examples of receiver verification measurements versus FEM temperature: (a) noise figure (NF) at $160 \mathrm{MHz}$ (the measurements include $6 \mathrm{~km}$ of SMF fiber between FEM and PREADU at ambient temperature) and (b) FEM power consumption. The causes which lead to the higher NF in the $1270 \mathrm{~nm}$ link are discussed in Sec. 3.2.1.

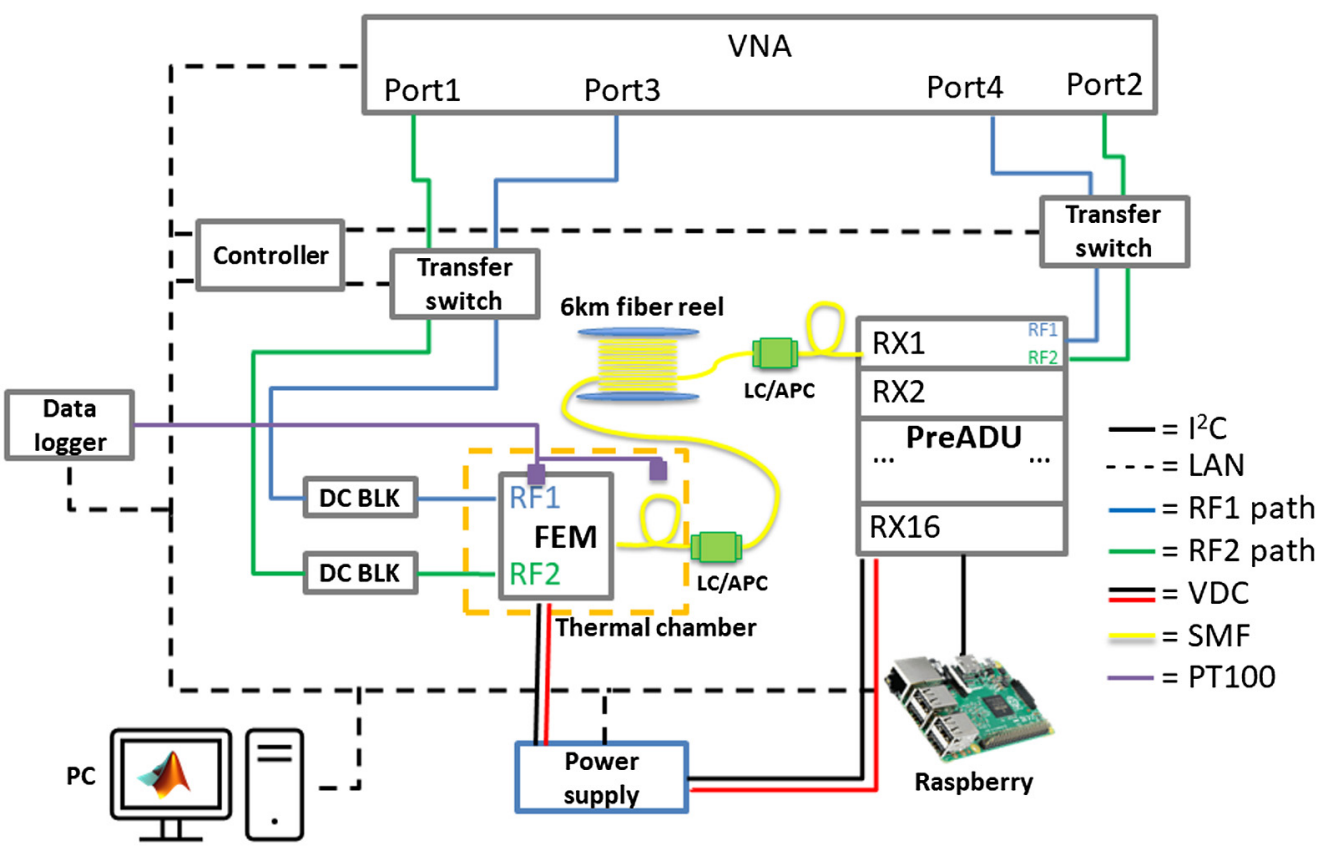

Fig. 12 Measurement setup to characterize the SKA-low receiver versus the FEM operative temperature. The Keysight PNA-X has been programmed in order to measure not only the four ports $S$-parameters but also the NF, the 1-dB compression point and the second- and third-order intercept points. A MATLAB code has been written in order to control all instrumentation, acquire, and process the data.

driving functions allows basing these adjustments on a combination of end-to-end measurements and modeled effects, reducing the dependence on real-time sky-based measurements, as well as allowing statistical analysis to improve the long-term behavior of the system.

The period over which the system must remain stable depends on both the science requirements for precision and the time scales of both the physical environmental variations and measurement techniques. Several different time scales emerge from these considerations: typical environmental effects show periodicity of 12 and $24 \mathrm{~h}$, local thermal time constants of electronic components are typically of order minutes, and on-sky measurements can be affected by ionospheric effects that suggest recalculation epochs of order $10 \mathrm{~min}(600 \mathrm{~s})$. A further complication results from the wide field of view of individual antennas, which effectively see the entire sky hemisphere and are thus dominated by large-scale features such as the galaxy and Sun but cannot resolve individual point sources. This means that in practice, direct interferometric calibration of individual antennas cannot be done to a sufficient level of accuracy if neither the galaxy or the 


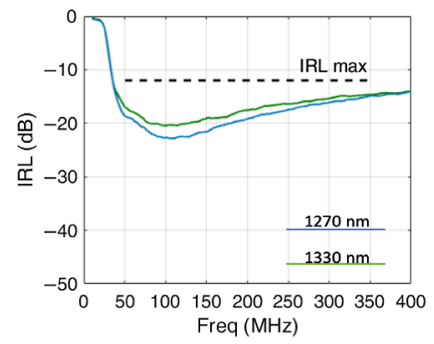

(a)

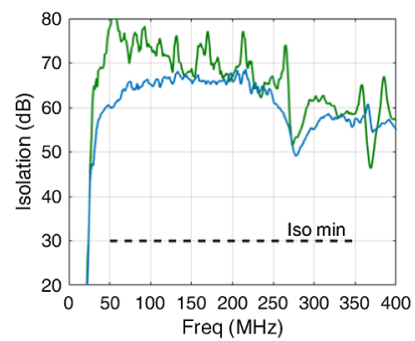

(d)

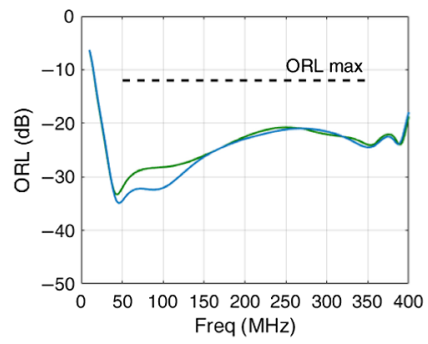

(b)

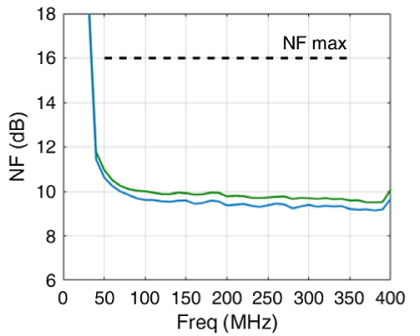

(e)

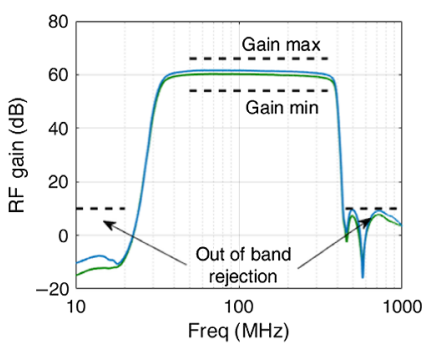

(c)

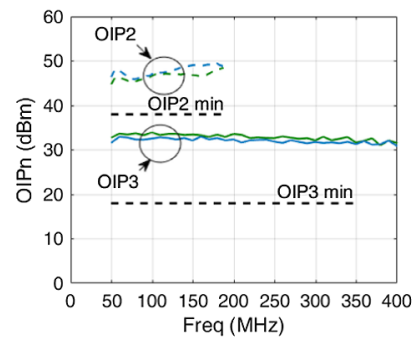

(f)

Fig. 13 (a)-(f) Example of FAT measurements of one FEM produced for AAVS2. The measurements are performed automatically with the setup as shown in Fig. 12 but with the FEM at ambient temperature and without any fiber reel between FEM and PREADU.

Sun is visible, limiting the time periods when such measurements can be made. The implications of these timescales are profound, as it is clear that a parameter that varies on 24-h timescales need not be recalculated frequently, while those that vary at the level of minutes cannot be corrected by measurements on a 10-min cadence. Therefore, a combination of calibration techniques including direct but infrequent on-sky measurement of signal chain gains with forward modeling of locally faster variations will be necessary to achieve the SKA requirements.

Analysis of the signal chain confirms that the dominant effect causing changes in the signal chain transfer functions (phase and gain) is driven by the environmental temperature of the components. The SKA system architecture locates most of these electronic components in the relatively stable environment of the processing facilities thus limiting this issue to the LNA, the FEM, and the optical fiber itself. In the following sections, the contributions of the FEM and optical fiber will be shown. The influence of the temperature variation on the LNA will not be considered as it is not part of the receiver. However, it has been verified that its contribution is not significant with the respect to the ones of the other components.

\subsubsection{Amplitude and phase stability due to the FEM temperature only}

To investigate the impact of the only temperature exposition of the FEM on overall stability, a characterization has been performed in the laboratory using a climatic chamber, where the temperature was varied from $-10^{\circ} \mathrm{C}$ to $+80^{\circ} \mathrm{C}$.

Figure 14 represents a measurement of a sample of six FEMs taken from the AAVS2 production, showing the variation of the absolute gain of both 1270- and 1330-nm channels. The curves decrease monotonically with temperature and justify, as shown in the previous section, why it was necessary to reduce as much as possible the FEM maximum operative temperature to $+65^{\circ} \mathrm{C}$ during the day. From the same figure, it is also possible to gather that by setting the correct value of the DSA, it is possible to obtain the gain requirement $(41 \mathrm{~dB})$ over the entire temperature range. The gain drop of the 1270-nm channel has been found to always be worse than the 1330-nm one for all measured devices also from different suppliers. The reason lies in the fact that the 1270-nm optical carrier is at $90 \mathrm{deg}$ with respect to the fiber optic and has to be reflected by the WDM thin film filter and not be transmitted as for the 1330-nm carrier, making it more sensitive to mechanical stress on the package assembly due to temperature variations. 


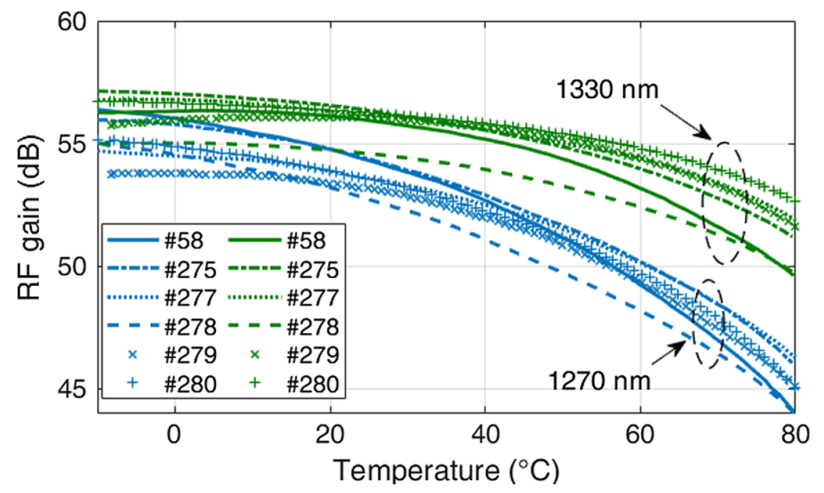

Fig. $14 \mathrm{RF}$ gain at $160 \mathrm{MHz}$ versus FEM operative temperature (with $6 \mathrm{~km}$ of G.652.D fiber and PREADU, with DSA set at $0 \mathrm{~dB}$ both at ambient temperature).

A study to reduce such difference between the two channels is undergoing by the suppliers of the optical components and new samples will be tested in the coming months.

Regarding the phase stability, Fig. 15 shows the impact of the RF electronics of the FEM when subjected to temperature variation. This figure shows the absolute phase variation of both wavelengths. These experimental results can be compared with the expected (simulated) ones generated by the combined effect of temperature-induced wavelength shift of the laser and fiber chromatic dispersion. By comparison, it is possible to appreciate the same order of magnitude of the absolute phase variation, which furthermore justifies the choice of the two wavelengths.

Considering that in SKA-low the temperature variations on the FEMs can be easily different one from the other due to the high dislocation of the antennas and that those cannot be thermalized since this would require a major increase of cost and power consumption, the phase error must be minimized as much as possible acting on the choice of the components.

Roughly speaking, it is possible to show that, neglecting the variation of the refractive index of the fiber with the temperature, the phase error among two optical links of length $L$, carrying an RF signal of frequency $f_{\mathrm{RF}}$ and working at same nominal wavelength $\lambda$, produced by the temperature variations on two separate transmitters TX1 and TX2 can be written as

$$
\delta \phi_{1}-\delta \phi_{2} \simeq 2 \pi \cdot f_{\mathrm{RF}} \cdot D \cdot C_{\lambda} \cdot L \cdot\left(\delta T_{\mathrm{TX} 1}-\delta T_{\mathrm{TX} 2}\right),
$$

where $\delta \phi_{1}, \delta \phi_{2}$ are the absolute values of phase drift due to the absolute temperature variation $\delta T_{\mathrm{TX} 1} \delta T_{\mathrm{TX} 2}$ on the transmitters, respectively. Moreover, $D$ is the fiber chromatic dispersion coefficient and $C_{\lambda}$ is the wavelength-temperature slope coefficient. This simple equation shows that the phase error is proportional to these last two quantities, which address the choice of the

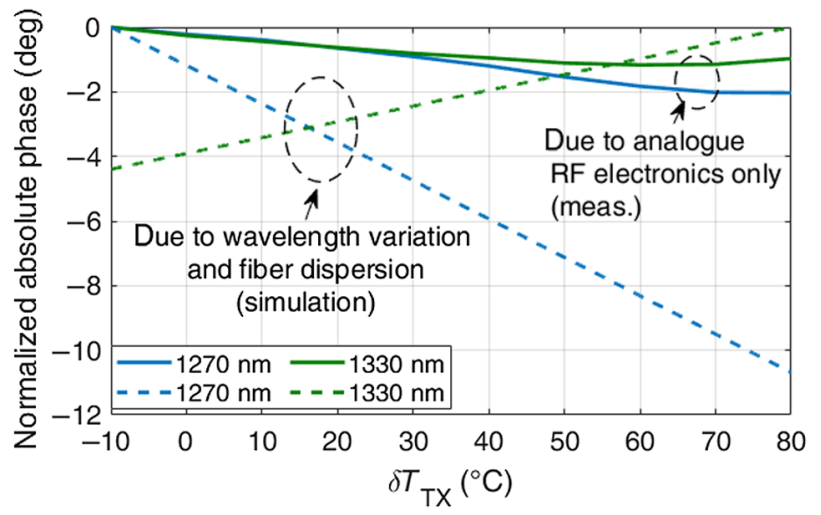

Fig. 15 Normalized absolute phase variation with respect to FEM operative temperature $\delta T_{\mathrm{TX}}$ normalized (to their max values) at $160 \mathrm{MHz}$ for both RF1 and RF2 considering only the analogue RF electronics (solid lines) and the one expected due to temperature-induced wavelength-shift and fiber dispersion (dashed lines). 
wavelengths to the ones that minimize chromatic dispersion and wavelength-temperature slope coefficient.

Among the dual-wavelength laser technologies available on the market, which requires at least $60 \mathrm{~nm}$ of spacing to let the C-WDM diplexer work fine, the selected optical wavelengths pair has been $1270 / 1330 \mathrm{~nm}$, which was preferred with respect to other options such as the one more often used in commercial applications, 1310/1550 nm.

Although the value of $C_{\lambda} \simeq 0.1\left(\mathrm{~nm} \mathrm{~K}^{-1}\right)$ is almost similar for the mentioned wavelengths, the chosen ones present values of chromatic dispersion around $D(\lambda=1270 \mathrm{~nm}) \simeq$ $-3\left(\mathrm{ps} \mathrm{nm}^{-1} \mathrm{Km}^{-1}\right)$ and $D(\lambda=1330 \mathrm{~nm}) \simeq 1.5\left(\mathrm{ps} \mathrm{nm}^{-1} \mathrm{Km}^{-1}\right)$. Instead, although at $1310 \mathrm{~nm}$ the dispersion is practically null, for $1550 \mathrm{~nm}$ the value of dispersion is about $D(\lambda=1550 \mathrm{~nm}) \simeq 17\left(\mathrm{ps} \mathrm{nm}^{-1} \mathrm{Km}^{-1}\right)$.

Figure 16 shows a simulation of the maximum phase variation obtained when two transmitters are subjected to a slight temperature difference for $6 \mathrm{~km}$ of G.652.D fiber. This figure compares $1310 / 1550 \mathrm{~nm}$ technology with the one employed at $1270 / 1330 \mathrm{~nm}$.

It is worth emphasizing that all the considerations and results above, which led to the choice of the transmitters' wavelengths, have been made neglecting the possible differences in the variation of the refractive index of the fibers due to external temperature, condition that can be easily achieved by burying the fiber cables. If this is not the case, and fibers are laid over the ground, the phase error is more influenced by the nonhomogenous temperature and solar radiation affecting the optical fiber, as will be shown later.

\subsubsection{Amplitude and phase stability due to the fiber temperature only}

A series of RFoF link measurements have been performed directly in the field at the MRO. Between 2014 and 2016, there had been several measurements on different fiber-optic cables. The measurements include several kilometer-length cables that are routed on the ground (and exposed to the temperature fluctuation), as well as those buried underground. We also performed measurements on two cables that are subsequently used for AAVS1 [the AFL spider web ribbon cables (SWR)] and for AAVS2 projects (the regular loose-tube fiber cable). Both AAVS cables are routed above ground in the field, as the MRO has shallow bedrock and it is challenging to dig a trench to bury the cable. The cables are routed along a 6-km distance between the AAVS site and the ASKAP control building, where the TPM are located.

We performed measurements to record the magnitude and phase fluctuations of the RFmodulated optical signal transported on the cables, to quantify the effect of the environmental exposure on the signal stability. The experimental setup is depicted in Fig. 17, utilizing the multiport vector network analyzer (VNA), the actual AAVS WDM RFoF modules, and different cables. In the experiments, we recorded the $S$-parameter (both magnitude and phase) of the RF signal from a pair of fiber-optic cores, selected from opposite extremities inside the cross section of the cable assembly. Each fiber carries the two wavelengths (1270 and $1330 \mathrm{~nm})$, so in

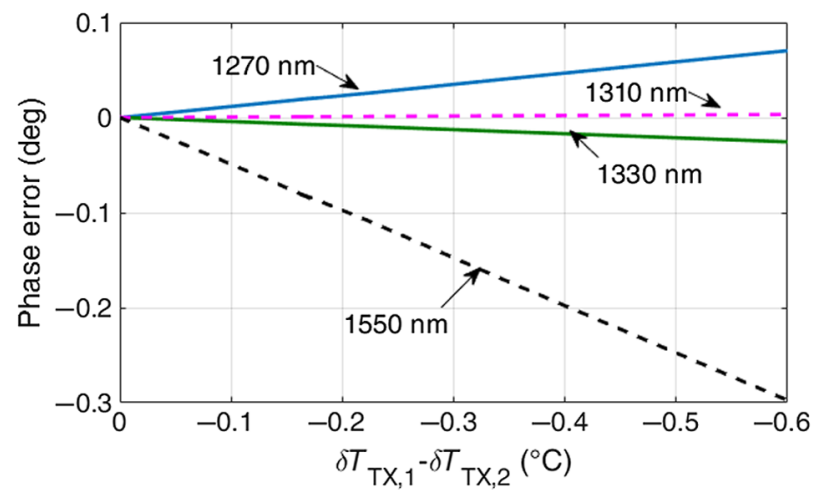

Fig. 16 Simulation of the phase error due to a different temperature behavior on the transmitters for 6-km RFoF link. In this figure, $\delta T_{\mathrm{TX} 1}$ and $\delta T_{\mathrm{TX} 2}$ represent the temperature of two different transmitters, and the curves are shown as a function of the difference of such temperatures. 


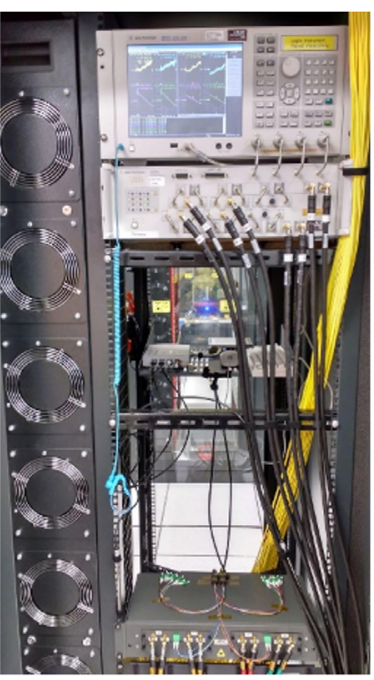

(a)

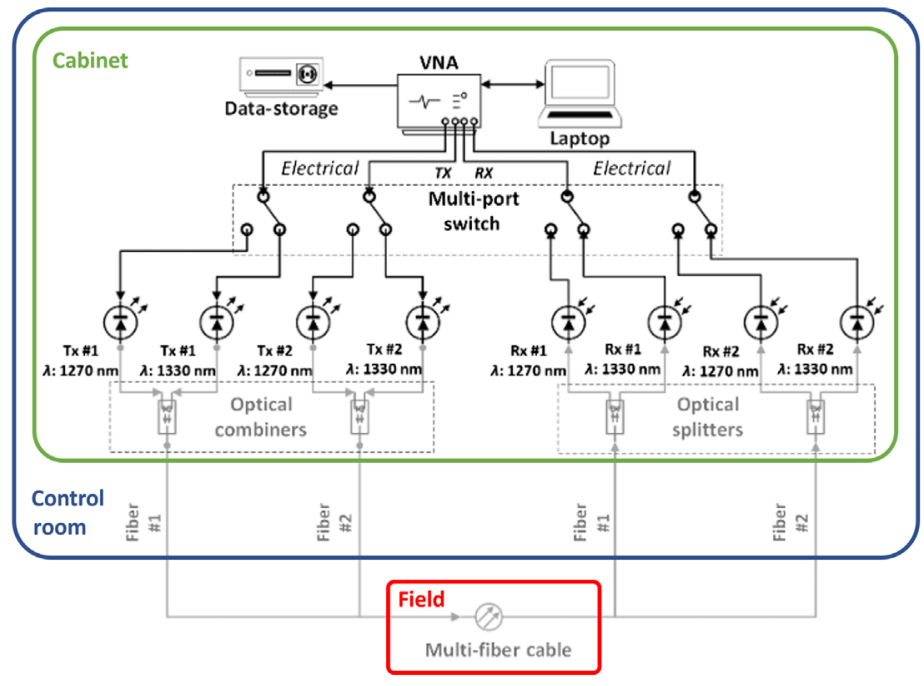

(b)

Fig. 17 (a) Experimental setup to measure the magnitude and (b) phase stability of RF-modulated signal transported through fiber-optic cable in the field, utilizing multiport VNA and AAVS RFoF modules.

total, there are four sets of $S$-parameter from a pair of RFoF modules. We performed a full 24-h measurement each day, with an interval of $10 \mathrm{~s}$ between consecutive measurements, across four VNA channels covering SKA-low frequency band (50 to $350 \mathrm{MHz}$ ) whenever possible, depending on the aperture settings of the VNA. This is to ensure accurate phase slope measurement across the frequency and that the phase difference between two adjacent measurement points is $<180 \mathrm{deg}$. The VNA and RFoF module were placed inside a temperature-controlled server rack at the control building to ensure stable operating temperature and minimal fluctuation contribution from the instruments and from the RFoF optical transmitter and receiver (OTX and ORX, respectively).

The results of the field test indicated that outdoor exposure mainly affects the phase stability of the RF signal transmitted over the FO cable. Indeed, for a pair of fibers, assuming that the linear thermal expansion coefficient and the temperature coefficient of refractive index of each fiber are similar, the phase error due to temperature fluctuation of signal transported in a pair of fiber cores $\left(F_{1}\right.$ and $\left.F_{2}\right)$ can be estimated as ${ }^{33}$

$$
\begin{aligned}
\delta \phi_{1}-\delta \phi_{2}= & 2 \pi \cdot f \cdot\left|\delta \tau_{1}-\delta \tau_{2}\right|=2 \pi \cdot f \cdot \mid\left[\left(n \cdot \alpha_{L}\right)+\beta_{n}\right] \\
& \cdot \frac{1}{c} \cdot\left[\left(L_{0, F_{1}} \cdot \delta T_{F_{1}}\right)-\left(L_{0, F_{2}} \cdot \delta T_{F_{2}}\right)\right] \mid,
\end{aligned}
$$

where $\delta \phi_{1}, \delta \phi_{2}$ are the absolute phase variations of the two links of initial lengths $L_{0, F_{1}}, L_{0, F_{2}}$, respectively, whereas $\delta \tau_{1}, \delta \tau_{2}$ are their correspondent group delay variations. Moreover, $\alpha_{L}=\frac{1}{L} \frac{d L}{d T}$ and $\beta_{n}=\frac{d n}{d T}$ are, respectively, the linear thermal expansion coefficient and the refractive index temperature coefficient, whereas $\delta T_{F_{1}}, \delta T_{F_{2}}$ are the temperature variations of fibers 1 and 2, respectively, $n=1.47$ is the refractive index of the fibers core, and $c$ is the speed of light in vacuum. Figure 18 shows an example of absolute phase variation (i.e., $\delta \phi_{1}$ or $\delta \phi_{2}$ ) for $6 \mathrm{~km}$ of fiber length and considering typical value for $\alpha \sim 0.56 \times 10^{-6}\left({ }^{\circ} \mathrm{C}^{-1}\right)$ and for $\beta \sim 1.2 \times 10^{-5}\left({ }^{\circ} \mathrm{C}^{-1}\right)$. This figure compares then Eqs. (1) and (2), showing that the main contribution to the absolute phase variation, and consequently on the phase error, is given by the variation of the refractive index with temperature where the latter produces a phase error higher by about two orders of magnitude.

Equation (2) gives us an estimate of the relative phase variation in a pair of fibers. Based on the measurement result in the field, this equation also gives us an estimate of the phase variation with respect to thermal expansion as we scale the length of the cable, assuming that the cable has the same property with the one used in the measurement. 


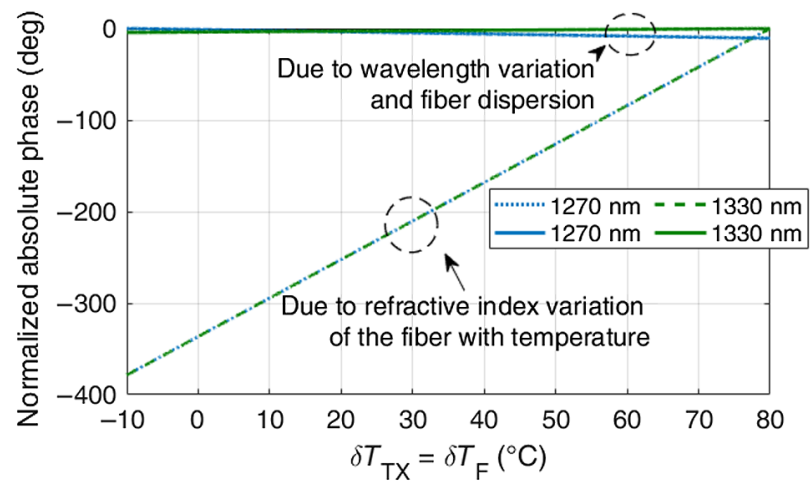

Fig. 18 Normalized absolute phase simulated considering the temperature-induced wavelengthshift on the FEM only (solid lines) and considering only temperature-induced fiber refractive index change (dashed lines).

Figure 19 shows the maximum relative magnitude variation (a) and relative phase variation (b) processed for each 600-s window in 24-h from two different RFoF modules, the first module operates at $1270 \mathrm{~nm}$ and the second module operates at $1330 \mathrm{~nm}$. It can be seen that thermal effects affect the phase stability more than the magnitude stability. Note that although the measurements were performed on a fiber link of $10.6 \mathrm{~km}$ length, the phase plots shown in Fig. 19(b) are scaled to $6 \mathrm{~km}$ to reflect the SKA-low transmission. Moreover, the relative phase variation at a particular frequency not covered by the measurement can be estimated by linear extrapolation of the data taken between 100 and $190 \mathrm{MHz}$.

The magnitude drift and relative phase drift over 24-h period are depicted in Figs. 20(a) and 20(b), where the maximum phase difference between two different fibers can reach up to $\sim 40 \mathrm{deg}$ (phase, on a cable with a total length of $6 \mathrm{~km}$ ), for RF signal transmitted at $160 \mathrm{MHz}$ across the cable. The maximum drift, relative to the start of the measurement taken at midnight, occurs around noon time, which corresponds with the maximum ambient temperature on site at the MRO. By analyzing the weather data [see Fig. 20(c)], we have established that the phase stability is sensitive to thermal fluctuation on the cable, which is correlated with the solar irradiation, ambient temperature data, and wind.

One possible option to minimize the temperature variation of the cable is by underground burial. We performed measurements of buried cable versus surface laid cable, where it was found from the measurement results that buried cable has very good gain and phase stability compared to the surface laid cable; ambient temperature, solar irradiation, or wind changes have negligible effect on the stability of the phase of the RF signal transmitted through the cable in the 600-s window throughout a 24-h period (Fig. 21). Moreover, as predicted by the simulations, the phase

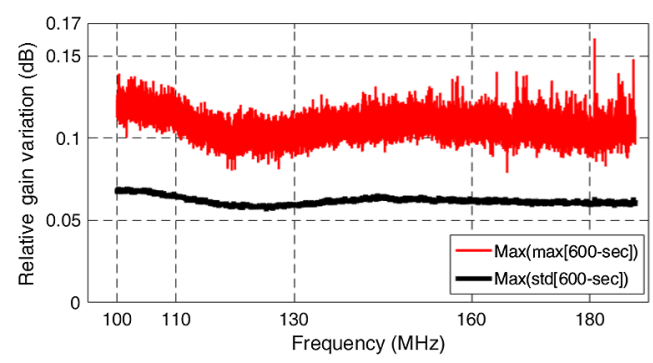

(a)

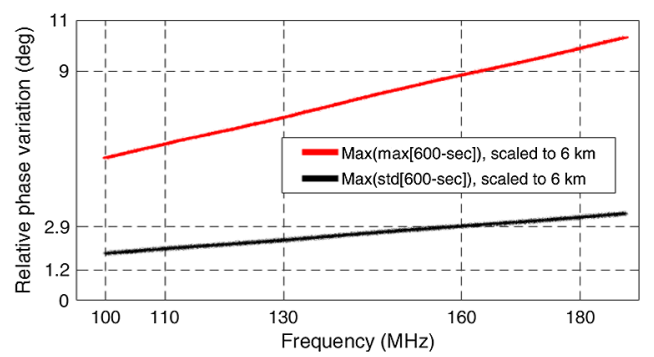

(b)

Fig. 19 (a) The 600-s relative magnitude variation statistics and (b) the 600-s relative phase variation statistics of RF signals (between 100 and $190 \mathrm{MHz}$ ) transmitted over a pair fiber-optic link over a 24-h period. The first module is operating at $1330 \mathrm{~nm}$ and the second module is operating at $1270 \mathrm{~nm}$, transmitted across two different fiber cores (worst-case scenario). The cable is a $\sim 5.3-\mathrm{km}$ loose tube cable used in AAVS2, and it is a loop-back transmission forming a total length of $\sim 10.6-\mathrm{km}$ transmission. The plot shown for relative phase variation statistics is scaled to $6 \mathrm{~km}$ to reflect the SKA-low transmission distance. 


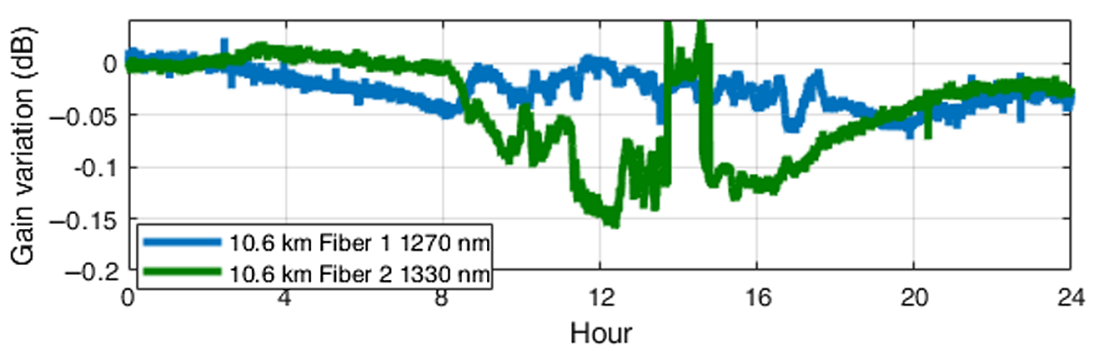

(a)

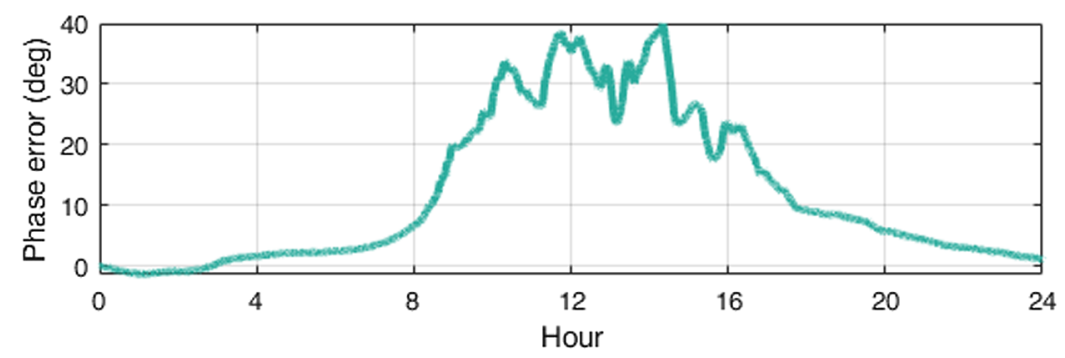

(b)

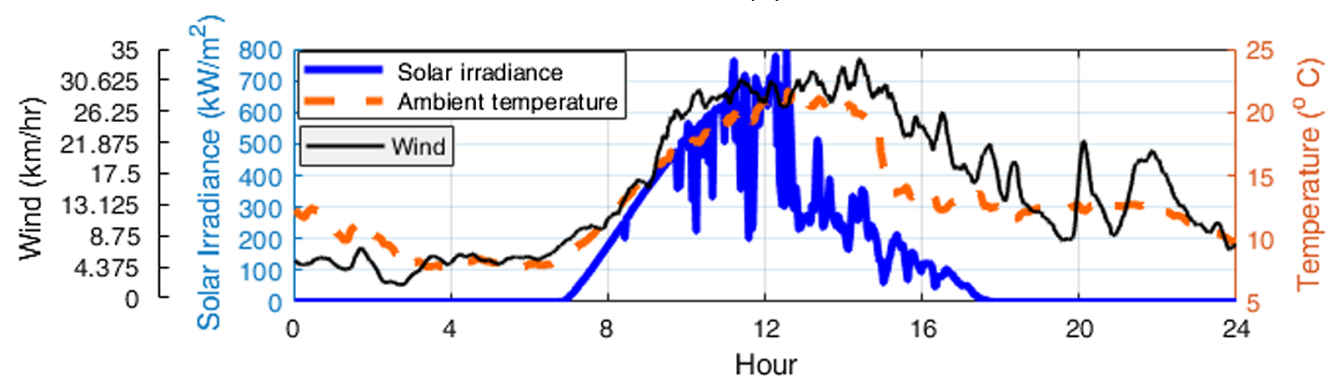

(c)

Fig. 20 (a) The 24-h magnitude drift and (b) the relative phase drift of RF signals (at $160 \mathrm{MHz}$ ) transmitted over a pair fiber-optic link where the first module is operating at $1270 \mathrm{~nm}$ and the second module is operating at $1330 \mathrm{~nm}$, the RF signal from each module is transmitted across two different fiber cores. The cable is a 5.5- $\mathrm{km}$ loose tube cable used in AAVS2, and it is a loopback transmission forming a total length of $11-\mathrm{km}$ transmission. The relative phase drift plot shown here is scaled for a 6-km transmission to reflect the SKA-low transmission distance. (c) The solar irradiation, ambient temperature, and wind speed are taken from the weather station data at the MRO.

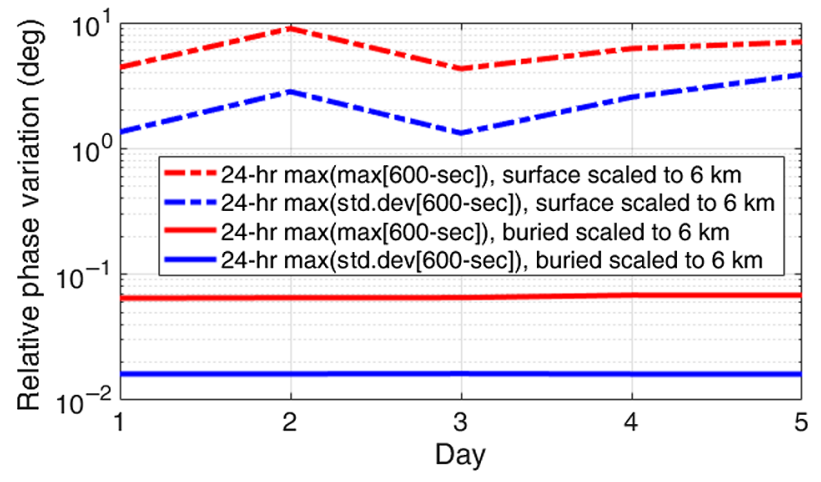

Fig. 21 The 5-day 600-s relative phase variations statistics of the 6-km surface laid cable (dashed line) versus 6-km buried cable (solid line) at $160 \mathrm{MHz}$. 
variations induced by the change with temperature of the fiber refractive index (surface laid cable) are of two orders of magnitude higher compared to the ones produced by the only temperature-induced wavelength shift on the FEM (buried cable).

As a result of the investigations performed on the AAVS and EDA demonstrators, which with a 5-km length of exposed optical fiber cable can be considered as worst-case examples, the fiber cables will largely be buried in the SKA-low system. The full range of temperature and solar irradiation changes will therefore be undergone only by short-fiber segments, up to $\sim 30 \mathrm{~m}$ long, within the antenna fields.

\subsection{Mitigation of the Detrimental Effects due to Rayleigh Backscattering}

During a test driven site trip on November 2018 (so called phase 0), a SKALA4-AL antenna and an EDA2 dipole were installed at MRO. The antennas were connected to a dedicated TPM hosted in the ASKAP control building through the fiber cable deployed for AAVS1. Since the first acquisitions, two problems have been encountered, related, respectively, to the presence of noise and to the presence of distortion terms.

- On both EDA2 and SKALA4 antennas, the noise floor was "jumping" and also some quite high peaks were appearing. Both weird effects were more pronounced at low frequency $(<50 \mathrm{MHz})$ but appreciable even in all SKA-low band 50 to $350 \mathrm{MHz}$.

- In addition to this, a counterintuitive behavior was exhibited during a laboratory characterization of the only RFoF link. Such behavior appeared in the same fashion for each of the two wavelengths 1330 and $1270 \mathrm{~nm}$, carrying the $Y$-polarized and the $X$-polarized RF signals received by the antenna, respectively. It consisted in the fact that in the evaluation of the intercept point of the second order (IP2) of the link, when the latter is directly modulated with a sinusoidal tone, the power of the second harmonic distortion term $\left(\mathrm{HD}_{2}\right)$ exhibited rather high values, which were unexpectedly increasing in correspondence to a reduction of the power of the modulating tone.

The cause of both problems has been identified in the Rayleigh optical backscattering. ${ }^{34}$ This effect is originated by the interaction of the emitted light by the laser and the back-reflected one by the fiber into the laser cavity itself, so basically it is strongly dependent from the optical isolator integrated in the optical source. The detriment is equally present on both the RFoF links used for $X$ - and $Y$-polarization signals because of the vicinity of the two wavelengths utilized (1270 and $1330 \mathrm{~nm}$, respectively).

The solution adopted to counteract the mentioned effects of Rayleigh backscattering (RBS) consisted in the direct modulation of the laser source through a sinusoidal tone of appropriate amplitude $I_{\text {dith }}$ and frequency $f_{\text {dith }}$ (see Fig. 22).

This solution (often indicated as dithering tone technique) was already proposed in the past, with the aim to reduce interferometric noise in optical fiber systems, including the RBS induced

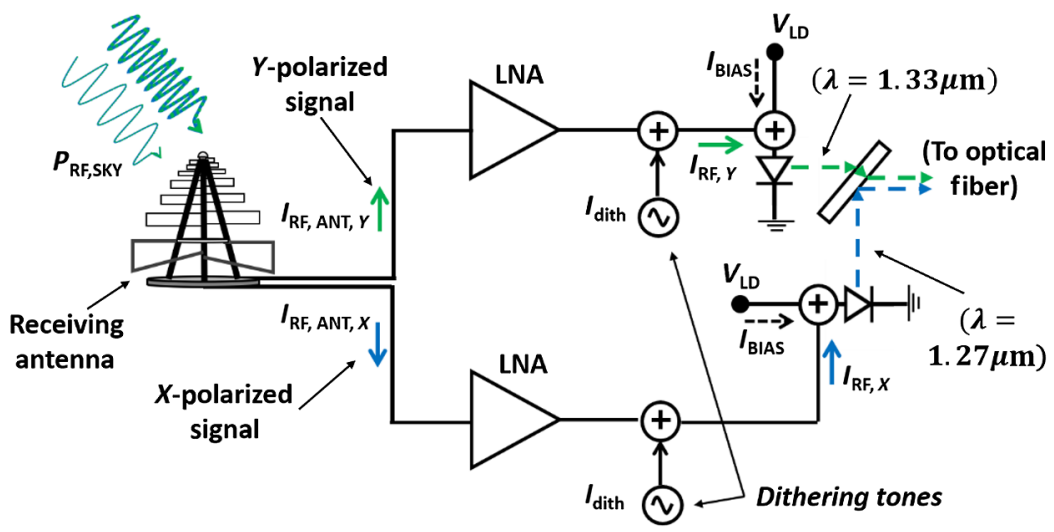

Fig. 22 Simplified scheme depicting the introduction of the dithering tones in each of the two RFoF transmitters utilized in the receiving chain of AAVS2. 
noise. ${ }^{35}$ Its beneficial effect in attaining the reduction of the noise level at low frequencies was then expected.

In addition to this, a comprehensive theoretical and experimental investigation was performed with reference to the counterintuitive behavior observed in the spurious frequencies generated at the receiving end of the RFoF system by a sinusoidal modulating tone. The reason of this behavior could then be explained, and thanks to this theoretical result it was possible to demonstrate that the same dithering tone technique could constitute an appropriate countermeasure also in reducing the global impact of the system nonlinearities.

Figure 23 visualizes the behavior of the RFoF system in terms of generation of $\mathrm{HD}_{2}$, which consists in the amount of power received at the double $\left(2 f_{\mathrm{RF}}\right)$ of the modulating tone frequency.

For values of $P_{\mathrm{RF}}$ higher than a certain threshold value (in the example of the figure around $0 \mathrm{dBm}$ ), the laser nonlinearity is the prevailing source of $\mathrm{HD}_{2}$, regardless of the frequency $f_{\mathrm{RF}}$ of the modulating tone. This gives rise to an "intuitive" behavior of the nonlinearities, since increasing values of the amplitude of the modulating current determine increasing values of $\mathrm{HD}_{2}$.

On the contrary, it can be appreciated that for a given value of modulating frequency $f_{\mathrm{RF}}$ belonging to the interval between $f_{\mathrm{RF}}=10 \mathrm{MHz}$ and $f_{\mathrm{RF}}$ a little $>350 \mathrm{MHz}$ (interval that includes the SKA-low band 50 to $350 \mathrm{MHz}$, when $P_{\mathrm{RF}}$ is progressively reduced from $0 \mathrm{dBm}$ to lower values, $\mathrm{HD}_{2}$ does not exhibit a monotonic behavior. Indeed, it initially decreases, then it "counterintuitively" increases again, reaching a maximum value (correspondent to the yellow region in Fig. 23), after which a final decrease of $\mathrm{HD}_{2}$ takes place.

The reason of this behavior lies in a combined effect of RBS and laser frequency chirp. As a consequence of that, the behavior of $\mathrm{HD}_{2}$ can be shown to be dependent on the phase modulation index $M_{\mathrm{RF}} \propto K_{f} \frac{\sqrt{P_{\mathrm{RF}}}}{f_{\mathrm{RF}}}$, where $K_{f}$ represents the adiabatic chirp coefficient of the laser utilized. ${ }^{36}$ Note that this phenomenon regards all the possible spurious frequency terms that can be received by the RFoF link.

As mentioned above, the dithering tone technique constitutes an effective countermeasure to the nonlinear spurious frequencies generation just described, as well as to the undesired presence of noise peaks and noise floor jumps that were observed mostly for $f_{\mathrm{RF}}<50 \mathrm{MHz}$ but were present also within the SKA-low bandwidth.

It is important to point out that to solve these issues, it was not possible to act on other causes (such as the insertion of a double-stage isolator to reduce the amount of RBS feedback and the

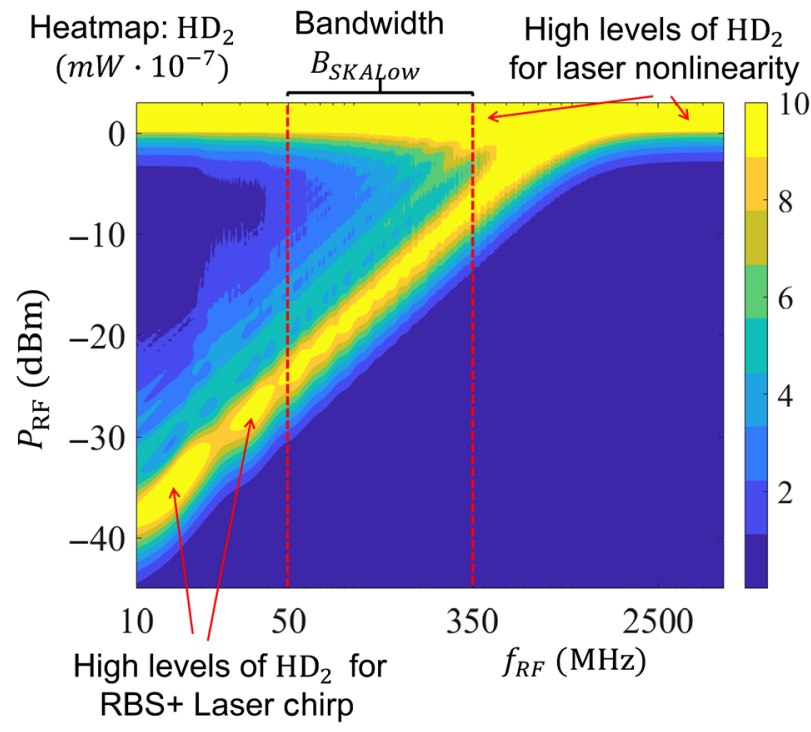

Fig. 23 Behavior of the power received by the RFoF system at the frequency $2 f_{\mathrm{RF}}$ where both the "intuitive" behavior $\left(P_{\mathrm{RF}} \geq 0 \mathrm{dBm}\right)$ and the "counterintuitive" one $\left(P_{\mathrm{RF}}<0 \mathrm{dBm}\right)$ can be appreciated. 
use of an external modulator to reduce the chirp coefficient $K_{f}$ ) since it would have increased dramatically the cost of the link.

Figures 24(a) and 24(b) allow to appreciate the beneficial effects to the quality of the signal received by the RFoF link in terms of reduction of the noise effect. The bandwidth 5 to $45 \mathrm{MHz}$ is represented, which is the one where it is possible to better appreciate the improvement coming from the dithering tone technique adoption. The spikes are clearly recognizable in the live spectrum yellow trace on the top of Fig. 24(a), whereas the jumps of the noise floors appear as horizontal stripes in the spectrogram, represented in the bottom part of this figure.

In Fig. 24(b), the effect of the introduction of the dithering tone in the system can be clearly appreciated, since the spikes and the horizontal stripes have disappeared, respectively, in the live spectrum and in the spectrogram.

In addition to the impact on spikes and noise, the effect of the dithering tone is shown in Fig. 25 for which regards the level of the output intercept point of second order (OIP2), where it can be appreciated the strong improvement at low frequency.

However, it is important to point out that the insertion of the dithering tone, which directly modulates the laser, can generate itself intermodulation distortion that must be controlled properly. To derive a specification of the maximum acceptable level of the intermodulation that can be tolerated, it is considered that its level should be below the noise at the output of the receiver considering the finest bandwidth of SKA-low $(266 \mathrm{~Hz})$. For example, considering the most powerful RFI (generated by the Orbcomm satellite) at $160 \mathrm{MHz}$ and considering the use of the SKALA4 antenna, an intermodulation product lower than at least $50 \mathrm{~dB}$ is required to make it not recognizable by the system.

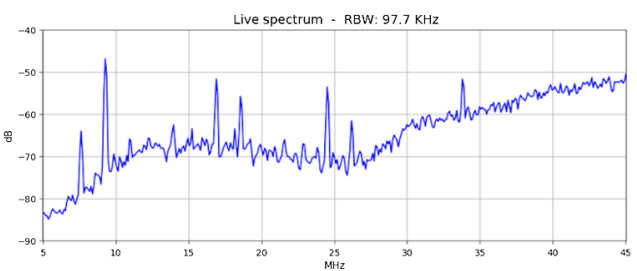

Spectrogram of TPM-18 RX-02 SKALA-4 Pol-Y 2019-01-10

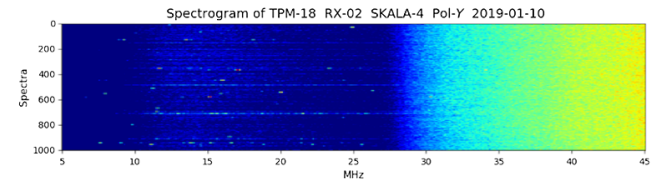

(a)

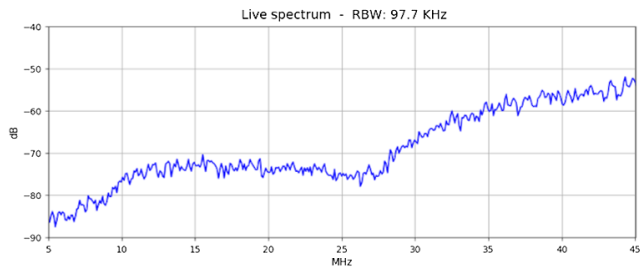

Spectrogram of TPM-18 RX-02 SKALA.40 POL-Y 2019-02-17

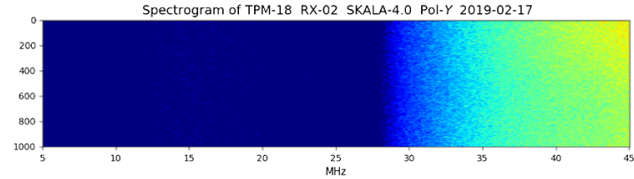

(b)

Fig. 24 Captured live image of the measured spectrum of the power received by the RFoF system in the interval 5 to $45 \mathrm{MHz}$ (a) with and (b) without the dithering technique applied.

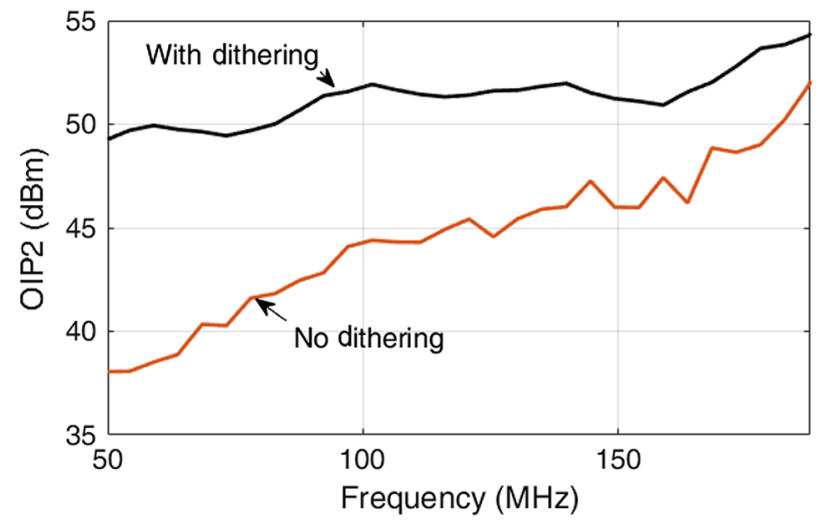

Fig. 25 Example of behavior of the OIP2 for the 1330-nm wavelength of the RFoF link employing $6 \mathrm{~km}$ of fiber span. 
As a consequence of this theoretical and experimental investigation, the 256 RFoF links of both EDA2 and AAVS2 were all equipped with a dithering tone generator at $8.5 \mathrm{kHz}$ and amplitude $1.25 \mathrm{~mA}$, which allows the receiving end to reach acceptable values of the noise floor and of the undesired spurious frequency terms generated by both RBS and the dithering tone itself. A comprehensive explanation of the theory that is behind the improvements obtained can be found in Refs. 37 and 38.

\section{Ongoing Activities}

As mentioned above, there is still room for further improvements of the SKA-low receiver in view of its ultimate realization. It has already been mentioned, for example, that the suppliers of the optical components intend to reduce the gain difference between the 1270-nm channel and the 1330-nm one, which is related to the architecture of the WDM thin film filter utilized at the OTX side. Other activities that are presently proceeding in this direction are described below.

\subsection{Minimization of the Detriments Due to the Polarization-Dependent Loss}

Unexpected fluctuations of the optical received powers at both wavelengths were observed and investigated on the deployed RFoF links of AAVS2 in March 2020 (see Fig. 26). The behavior appeared to be connected to the exposure of the optical fiber cable to external environmental quantities (e.g., temperature). An investigation was then carried out at laboratory level, with the aid of a calibrated thermal chamber, to identify the reason of such behavior and envisage possible countermeasures.

The described undesired behavior resulted due to a combined effect of polarization mode dispersion (PMD) of the optical fiber and the polarization-dependent loss (PDL) of the WDM dichroic filter located at the receiver site, given by the polarization-dependent values of its reflection and transmission coefficients. The representation reported in Fig. 27 will be utilized to illustrate the mechanism causing the received RF power fluctuations.

As mentioned above, the two RF currents that come from the antenna downlink and carry the $Y$ - and $X$-polarized sky signals modulate, respectively, lasers emitting at wavelengths 1330 and $1270 \mathrm{~nm}$. The two resulting optical signals are multiplexed through a parallelepiped-like dichroic WDM combiner, which is hit by both fields with an incident angle of about 45 deg with respect to the vectors perpendicular to the respective incidence surfaces.

At the optical transmitters' site, the unavoidable temperature excursions undergone by the be transmitters can cause variations of parameters like the conversion efficiency of the lasers or the precise value of the wavelength of the emitted fields, whereas the polarization of the optical

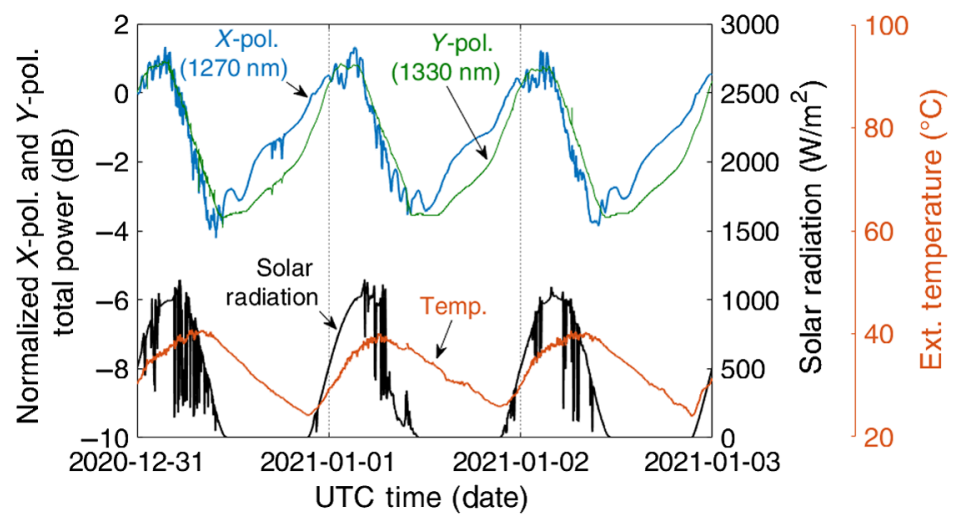

Fig. 26 Three days of on-site measurements of the total RF power at $160 \mathrm{MHz}$ (left axis) of both $X$ and $Y$ polarization (normalized at their respective first value) captured from one AAVS2 antenna, carried to the TPM by the designed RFoF link of about $6 \mathrm{~km}$ of fiber laid on the ground. Measurements of solar radiation and temperature taken from the Commonwealth Scientific and Industrial Research Organization (CSIRO) weather station are also shown in the right axes in order to correlate the behavior of the total RF power with the climatic conditions. 


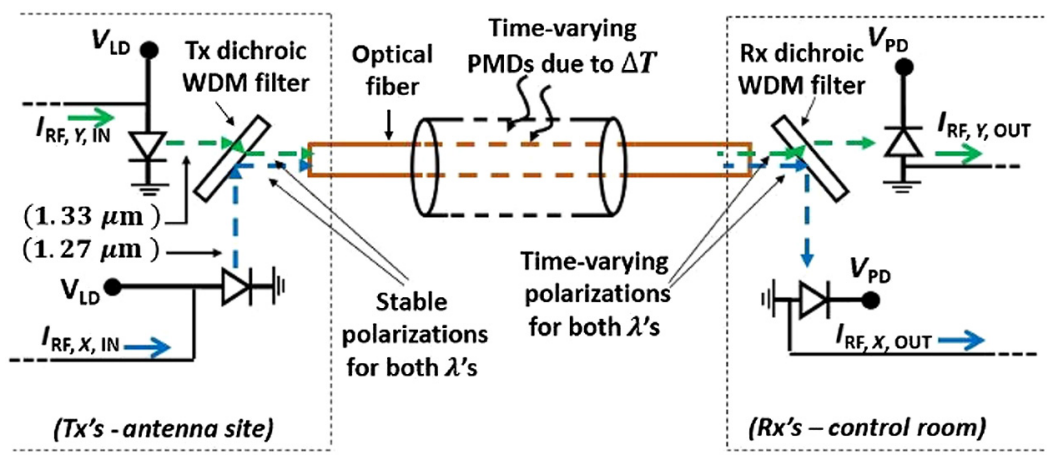

Fig. 27 Simplified representation of the mechanism determining the fluctuations of both the received RF powers carried by the optical fields at 1330 and $1270 \mathrm{~nm}$. PMD, polarization mode dispersion; Tx's, optical transmitters; and Rx's, optical receivers.

signals exiting from each of the two lasers (TE or TM or a combination of the two) can be regarded as stable in time. This fact brings as a consequence that the unavoidable difference exhibited by any dichroic filter presents in its reflection and transmission coefficients in dependence on the fact that the incident field exhibits a TE or a TM polarization does not determine any undesired fluctuation on the optical powers entering the optical fiber. Indeed, in passing through the dichroic WDM filter located at the transmitters' site, the optical field at $1330 \mathrm{~nm}$ (green dashed arrow in Fig. 27) will see an equivalent transmission coefficient stable in time, and stable in time as well will be the equivalent reflection coefficient seen by the optical field at $1270 \mathrm{~nm}$ (blue dashed arrow in Fig. 27).

Unlike what has just been described, each one of the two optical fields (the one at $1330 \mathrm{~nm}$ and the one at $1270 \mathrm{~nm}$ ) that hit the parallelepiped-like dichroic WDM splitter at the receiver side (see again Fig. 27) presents a polarization that changes in time. The reason for this behavior comes from the time variations of environmental quantities along the fiber path, which in turn induce time variations of the refractive index in different portions of the optical cable. The polarization dependence of the reflection and transmission coefficients of the dichroic filter determines this time undesired fluctuations of the optical powers detected by the two photodiodes.

In order to quantify the impact of the phenomenon, a laboratory test was performed on the RFoF system. The corresponding experimental setup is depicted in Fig. 28.
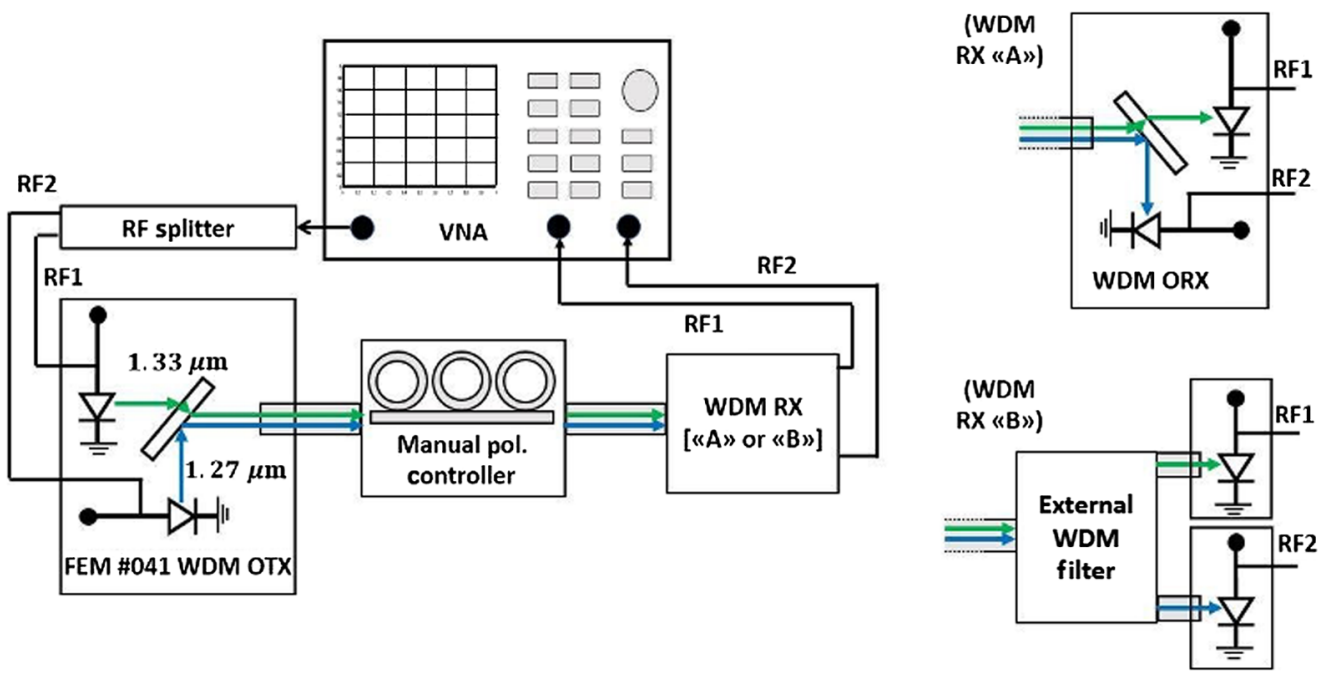

Fig. 28 Experimental setup utilized to determine the impact of the PDL caused by the characteristics of the optical receiver utilized. WDM RX "A:" optical receiver presently utilized in the RFoF system. WDM RX "B:" optical receiver where an external WDM filter with low polarization dependence separates the two wavelengths, which subsequently reach two separate PIN-based optical receivers. 


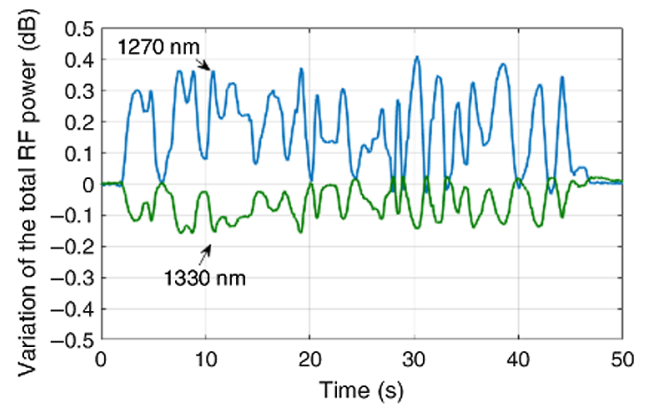

(a)

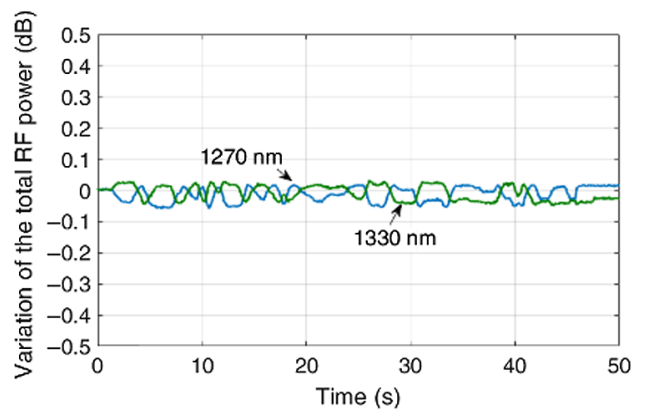

(b)

Fig. 29 Measured impact of the PDL on the RF total power caused by the (a) characteristics of the optical receiver presently adopted in the AAVS2 RFoF system and (b) characteristics of the optical receiver where an external WDM filter with low polarization dependence separates the two wavelengths, which subsequently reach two separate PIN-based optical receivers.

The results of the measurement of the total RF power performed utilizing the optical receiver presently adopted in the RFoF system are shown in Fig. 29(a), whereas the results of the measurement performed utilizing the optical receiver identified as WDM RX "B" in Fig. 28 are instead shown in Fig. 29(b).

From Figs. 29(a) and 29(b), the PDL of the two WDM filters can be computed from its relation with the variation of the RF power as follows:

$$
\mathrm{PDL}_{\mathrm{dB}}=\frac{1}{2} \cdot\left[\max (\text { total RF power variation })_{\mathrm{dB}}-\min (\text { total } \mathrm{RF} \text { power variation })_{\mathrm{dB}}\right] \text {, }
$$

where the factor $1 / 2$ takes into account the squared relation between received optical and electrical power. ${ }^{39}$

In case of the internal WDM filter, the PDL is about 0.2 and $0.07 \mathrm{~dB}$ for 1270 and $1330 \mathrm{~nm}$, respectively, while those values are greatly reduced with the external WDM filter to $0.04 \mathrm{~dB}$ for both 1270 and $1330 \mathrm{~nm}$.

With regard to this point, however, it must be noted that besides the possible improvements connected to a change in the receiving WDM filter characteristics, a significant mitigation of the PDL impact is expected to come from the operation of burying the optical cable, thanks to the resulting important reduction of the temperature fluctuations that would be undergone by the optical fiber in this case. The investigation activity is then proceeding in different directions, with the aim to identify the most appropriate measure to take in order to reach a satisfactory PDL mitigation.

\subsection{Monitoring of Fiber-Induced Delay on the RFoF Channel}

Ensuring the stability of the analogue signal transport from the antennas to the digitizers is essential to the performance of the SKA system. A major element of this is contributed by variability in the time taken by the signal to cover the distance to the digitizer. To address this issue, work has explored providing continuous monitoring of the fiber-induced delay in order to perform, when necessary, appropriate recalibrations of the phase differences among the contributions to the received signal coming from the different antennas. Factors that may affect the value of the fiber-induced delay include environmental variations of physical parameters (like temperature), which in turn present an impact on the behaviors of optical devices and components, such as the laser source and the optical fibers.

Within this context, a technique is presently investigated to monitor the variations in the time taken by the signal to cover the fiber length within the RFoF system. The corresponding system is represented in Fig. 30.

As can be appreciated, the system monitors the variation of the frequency amplitude response $\left(\left|S_{21}\right|^{2}\right)$ produced by a Michelson-type interferometer. A $\left|S_{21}\right|^{2}$ evaluator (which can either consist in a VNA or in a simpler device of much lower cost) modulates a 10-mW $1550 \mathrm{~nm}$ DFB laser 


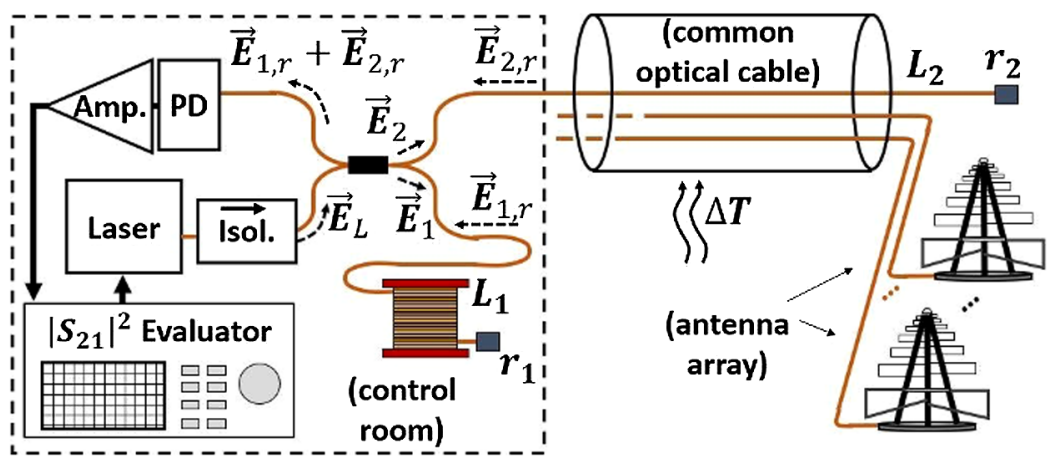

Fig. 30 Sketch of the proposed system in its possible implementation within the actual receiver of AAVS2, (PD, photodiode; Isol., optical isolator; and Amp., electrical amplifier).

with a sinusoidal RF tone of frequency $f_{\mathrm{RF}}$. The resulting optical signal then enters the mentioned interferometer, which is realized by exploiting a 50:50 fiber coupler. Still referring to Fig. 30, the left-hand side of the coupler is fed by the DFB laser, then one output of the coupler is connected to a reference thermalized fiber of length $L_{1}$, whereas the other output is connected to the fiber under test (FUT) of length $L_{2}$. Both fibers are terminated with a PC connector and the relative length between them should stay in the order of a few meters. The two reflected signals $\overrightarrow{E_{1, r}}, \overrightarrow{E_{2, r}}$ are then combined by the coupler and sent to a PIN photodiode, followed by an LNA of $42 \mathrm{~dB}$ of gain, where the observed behavior of $\left|S_{21}\right|^{2}$ is of the following type:

$$
\left|S_{21}\right|^{2} \propto\left|\cos \left(2 \pi f_{\mathrm{RF}} \Delta \tau\right)\right|^{2}
$$

where $\Delta \tau=\tau_{2}-\tau_{1}$ represents the delay difference between the two fibers. $\left|S_{21}\right|^{2}$ is therefore a periodic function having the minima located at $f_{\mathrm{RF}, \min , k}=\frac{(2 k+1)}{4 \Delta \pi}$, with $k=0,1,2, \ldots$ If the FUT is perturbed, then a variation of $\tau_{2}$ and correspondingly a variation $\Delta \tau$ are induced, which make the value of each $f_{\mathrm{RF}, \mathrm{min}, k}$ change correspondingly. Therefore, tracking one of the $k^{\prime}$ th $f_{\mathrm{RF}, \min }$ results in tracking the variation of $\tau_{2}$.

Note that while the proposed monitoring system is envisaged for application within SKAlow, its realizations have so far been limited to laboratory prototypes. In Figs. 31(a) and 31(b), the results of one of the experiments are shown where two fibers with nominal length of around $6 \mathrm{~km}$ have been employed, which is considered, up to now, the maximum fiber length deployable in SKA-low.

In particular, with a commercial optical time domain reflectometer system, the length difference between the two fibers resulted to be around $150 \mathrm{~m}$.

The chamber has been set to apply a temperature variation from $-10^{\circ} \mathrm{C}$ to $+50^{\circ} \mathrm{C}$ with a slope of $\frac{d T}{d t}=0.2^{\circ} \mathrm{C} / \mathrm{min}$. The temperature was measured with a resistance temperature detector placed within the chamber. The slope of the curve, normalized to the nominal length $L_{2}$, is about

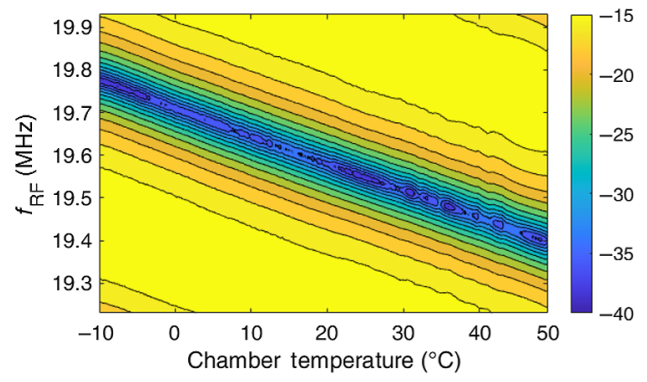

(a)

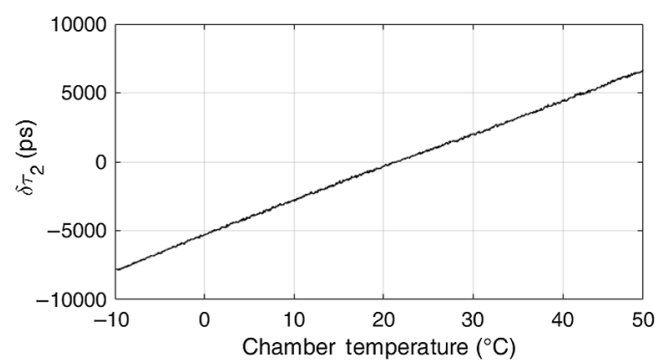

(b)

Fig. 31 (a) Shift of the minimum (blue region) of $\left|S_{21}\right|^{2}$ due to temperature variation inside the chamber. (b) Resulting measurement of the variation of $\tau_{2}$, named $\delta \tau_{2}$. 
$39 \mathrm{ps}\left(\mathrm{km} /{ }^{\circ} \mathrm{C}\right)$, in agreement with the theoretical expected value of $40 \mathrm{ps}\left(\mathrm{km} /{ }^{\circ} \mathrm{C}\right)($ see Ref. 33), showing a standard deviation about $60 \mathrm{ps}$, representing the precision of the system.

Further upgrades of the system are under study; for example, the possibility of using commercial reflectors to enhance the reflected power in both fibers and the possible replacement of the VNA with lower-cost devices.

\section{Conclusions}

The RFoF technology has been successfully applied to radio astronomy receiving systems for decades. But its application in SKA-low required intensive work to meet the requested RF performances considering very tight constraints, like the extreme environmental conditions of the Western Australian desert, the expected lifetime, and the low cost. The final baseline array layout, adopting a large CPF and several RPF, has evolved along with the progress made due the efforts in RFoF technology evaluation that started more than 10 years ago, coordinated by INAF with the support of other international partners. Extensive laboratory tests and several field campaigns at the MRO with the array demonstrators resulted in an RFoF receiver compliance with the SKA-low requirements. A custom designed system based on the dithering technique allows to counteract the effects of the Rayleigh optical backscattering which is particularly pronounced in the RFoF link for SKA-low due to the peculiar combination of the frequency band and power levels of the received signals. Nowadays, the RFoF SKA-low receiver is ready to be industrialized for the forthcoming mass production. The work still ongoing is targeted to better understand and eventually minimize the effect of the PMD/PDL.

\section{Acknowledgments}

This activity has been carried out in collaboration with Peter Maat, Pieter Benthem (ASTRON), Nima Razavi (UCAM), Adrian Suntijo (ICRAR), and all the members of the AADC Consortium established to develop the low-frequency aperture array element of SKA. The authors would like to underline that all the developing activities of the RFoF links have been made possible thanks to the fruitful collaboration and full support of the Italian industries Optel and Protech, in particular Eng. Franco Ferraresi and Eng. Enrico Lenzi.

\section{References}

1. "SKA telescope public website," https://www.skatelescope.org/ (2022).

2. P. E. Dewdney, "SKA1 design baseline description," SKA-TEL-SKO-DD-001, on behalf of SKAO (2013).

3. P. Benthem et al., "The aperture array verification system 1: system overview and early commissioning results," Astron. Astrophys. 655, A5 (2021).

4. A. J. J. van Es et al., "A prototype model for evaluating SKA-LOW station calibration," Proc. SPIE 11445, 1144589 (2020).

5. R. Wayth et al., "The engineering development array: a low frequency radio telescope utilising SKA precursor technology," Publ. Astron. Soc. Aust. 34, e034 (2017).

6. R. Wayth et al., "The engineering development array 2: design, performance and lessons from an SKA-low prototype station," J. Astron. Telesc. Instrum. Syst. 8(1), 011010 (2022).

7. "MWA telescope public website," https://www.mwatelescope.org/ (2021).

8. "ASKAP home page," https://www.atnf.csiro.au/projects/askap/index.html (2021).

9. F. Perini, "Analogue optical links experiences in the framework of the SKA/BEST activities," in Wide Field Astron. and Technol. Square Kilometre Array, Vol. 132 (2009).

10. A. Orfei and F. Perini, "Sostituzione dei coassiali di frequenza intermedia con link fibra ottica e caratterizzazione dei relativi segnali sull' antenna 32m di Medicina," IRA 472/13 Internal Report (in Italian), https://www.ira.inaf.it/Library/rapp-int/472-13.pdf.

11. P. E. Dewdney et al., "The Square Kilometre Array," Proc. IEEE 97(8), 1482-1496 (2009).

12. R. McCool et al., "Enhancing the sensitivity of radio telescopes using fiber-optic networks," URSI Radio Sci. Bull. 2006(317), 9-18 (2006). 
13. De Boer, "The Allen telescope array as prototype for the SKA," in IEEE Int. Symp. Antennas and Propagation and USNC/URSI Natl. Radio Sci. Meeting, Columbus, OH (2003).

14. S. Montebugnoli et al., "Large antenna array remoting using radio-over-fiber techniques for radio astronomical application," Microw. Opt. Technol. Lett. 46, 48-54 (2005).

15. J. Mena et al., "A radio-frequency-over-fiber link for large-array radio astronomy applications," J. Instrum. 8(10), T10003-T10003 (2013).

16. D. Crichton et al., "The hydrogen intensity and real-time analysis eXperiment: 256-element array status and overview," arXiv:2109.13755.

17. G. Swarup et al., "The giant metre-wave radio telescope," Curr. Sci. 60(2), 95-105 (1991).

18. N. Fagnoni et al., "Electrical and electromagnetic co-simulations of the HERA phase I receiver system including the effects of mutual coupling, and impact on the EOR window," arXiv:1908.02383v1 (2019).

19. M. P. Van Haarlem et al., "LOFAR: the low-frequency array," Astron. Astrophys. 556, A2 (2013).

20. S. J. Tingay et al. "The Murchison widefield array: the square kilometre array precursor at low radio frequencies," Publ. Astron. Soc. Aust. 30, 1-21 (2013).

21. G. Hallinan et al., "Monitoring all the sky all the time with the Owens Valley Long Wavelength Array," in Proc. Am. Astron. Soc. Meeting Abstracts, Vol. 225 (2015).

22. A. J. Faulkner and J. G. B. de Vaate, "SKA low frequency aperture array," in Proc. IEEE Int. Symp. Antennas Propag. USNC/URSI Nat. Radio Sci. Meeting, pp. 1368-1369 (2015).

23. J. Nanni et al., "VCSEL-based radio-over-G652 fiber system for short-/medium-range MFH solutions," J. Lightwave Technol. 36(19), 4430-4437 (2018).

24. J. Nanni et al., "Modal noise mitigation in 850-nm VCSEL-based transmission systems over single-mode fiber," IEEE Trans. Microw. Theory Tech. 64(10), 3342-3350 (2016).

25. J. Nanni et al., "Chirp evaluation of 850-nm single mode VCSEL exploiting modal noise in standard single mode fiber," IEEE J. Quantum Electron. 54(4), 1-11 (2018).

26. K. Y. Lau et al., "Signal-induced noise in fiber-optic links using directly modulated FabryPerot and distributed-feedback LASER diodes," J. Lightwave Technol. 11(7), 1216-1225 (1993).

27. A. Takemoto et al., "Distributed feedback LASER diode and module for CATV systems," IEEE J. Sel. Areas Commun. 8(7), 1359-1364 (1990).

28. "ASTRON public website," https://www.astron.nl/ (2022).

29. "ICRAR public website," https://www.icrar.org/ (2022).

30. "UCAM public website," https://www.cam.ac.uk/ (2022).

31. "INAF public website," www.inaf.it (2022).

32. "UNIBO public website," https://www.unibo.it (2022).

33. M. Bousonville and J. Rausch, "Velocity of signal delay changes in fibre cables," in Proc. DIPAC, Basel, Switzerland (2009).

34. J. Nanni et al., "Challenges due to rayleigh backscattering in radio over fibre links for the square kilometre array radio-telescope," in 21st Int. Conf. Transp. Opt. Networks, pp. 1-4 (2019).

35. J. A. Lazaro et al., "Rayleigh scattering reduction by means of optical frequency dithering in passive optical networks with remotely seeded ONUs," IEEE Photonics Technol. Lett. 19(1), 64-66 (2007).

36. J. Nanni et al., "Chirp evaluation of semiconductor DFB LASERs through a simple interferometry-based (IB) technique," Appl. Opt. 55, 7788-7795 (2016).

37. J. Nanni et al., "Controlling Rayleigh-backscattering-induced distortion in radio over fiber systems for radioastronomic applications," J. Lightwave Technol. 38(19), 5393-5405 (2020).

38. J. Nanni et al., "Optimum mitigation of distortion induced by Rayleigh backscattering in radio-over-fiber links for the square kilometer array radio-telescope," in Int. Top. Meeting Microwave Photonics, pp. 1-4 (2019).

39. C. H. Cox, III, "Analog optical links: theory and practice," Cambridge University Press (2004). 
Federico Perini received his Laurea degree in telecommunications engineering from the University of Bologna in 2001. Since 2002, he has worked for IRA/INAF at the Medicina Radio Observatory. His expertise covers LNA, RF/IF receivers, and RFoF systems designed and developed for the Italian Radio Telescopes (Northern Cross Array, VLBI dishes of Medicina and Noto, and the Sardinia Radio Telescope) and for international low-frequency instruments as LOFAR2.0 and the forthcoming SKA-Low.

Biographies of the other authors are not available. 